\title{
Regularity results for parabolic systems related to a class of non-Newtonian fluids
}

\section{Résultats de régularité pour des systèmes paraboliques liés à une classe de fluides non Newtoniens}

\author{
E. Acerbi ${ }^{a, *}$, G. Mingione ${ }^{a}$, G.A. Seregin ${ }^{b}$ \\ a Dipartimento di Matematica, Università, via M. D'Azeglio 85/a, 43100, Parma, Italy \\ b Steklov Mathematical Institute, St. Petersburg Branch, 27, Fontanka, 191011, St. Petersburg, Russia \\ Received 28 October 2002; accepted 26 November 2002
}

\begin{abstract}
We consider a class of parabolic systems of the type:

$$
u_{t}-\operatorname{div} a(x, t, D u)=0
$$

where the vector field $a(x, t, F)$ exhibits non-standard growth conditions. These systems arise when studying certain classes of non-Newtonian fluids such as electrorheological fluids or fluids with viscosity depending on the temperature. For properly defined weak solutions to such systems, we prove various regularity properties: higher integrability, higher differentiability, partial regularity of the spatial gradient, estimates for the (parabolic) Hausdorff dimension of the singular set.
\end{abstract}

(C) 2004 L'Association Publications de l'Institut Henri Poincaré. Published by Elsevier B.V. All rights reserved

\section{Résumé}

Nous étudions une classe de systèmes paraboliques du type :

$$
u_{t}-\operatorname{div} a(x, t, D u)=0
$$

où le champ vectoriel $a(x, t, F)$ possède des conditions de croissance non standard. Ces systèmes se présentent dans l'étude de certaines classes de fluides non Newtoniens comme les fluides electro-rhéologiques ou les fluides dont la viscosité dépend de la température. Nous prouvons différentes propriétés de régularité pour des solutions faibles convenables de tels systémes : intégrabilité et différentiabilité améliorées, régularité partielle du gradient spatial et estimations pour la dimension de Hausdorff (parabolique) de l'ensemble des points singuliers.

(C) 2004 L'Association Publications de l'Institut Henri Poincaré. Published by Elsevier B.V. All rights reserved

\footnotetext{
* Corresponding author.

E-mail addresses: emilio.acerbi@unipr.it (E. Acerbi), giuseppe.mingione@unipr.it (G. Mingione), seregin@pdmi.ras.ru (G.A. Seregin).
} 


\section{Introduction}

In recent years considerable attention was paid to the mathematical modelling of non-Newtonian fluids. These are fluids described by a set of equations including a stress tensor depending in a non-linear way by the gradient of the velocity. One of the first mathematical investigations of such models was carried out by Ladyzhenskaya in 1966 (see [17-19]); she considered the following system of equations that are known today as modified Navier-Stokes equations:

$$
\left\{\begin{array}{l}
u_{t}-\operatorname{div} a(\mathrm{D}(u))+D \pi=-\operatorname{div}(u \otimes u)+f, \quad \text { in } \Omega \times(0, T) \\
\operatorname{div} u=0,
\end{array}\right.
$$

where $\mathrm{D}(u)$ denotes the symmetric part of the gradient $D u$ and $\pi$ the pressure. The main point in the previous system, as just mentioned, is that the monotone vector field $a: \mathbb{R}^{9} \rightarrow \mathbb{R}^{9}$ depends in a non-linear way by $\mathrm{D}(u)$ :

$$
a(\mathrm{D}(u)) \approx\left(1+|\mathrm{D}(u)|^{2}\right)^{\frac{p-2}{2}} \mathrm{D}(u)+\text { terms with a similar growth }
$$

where $p>1$. The basic analysis of such systems goes back to Ladyzhenskaya and J.L. Lions (see also [23]). In particular, Ladyzhenskaya was also able to prove an existence and uniqueness theorem provided $p>2$ belongs to a certain range. Note that to find a set of equations for which uniqueness held was actually her main initial motivation; indeed, when studying systems as (1.1), she was not thinking of non-Newtoninan fluids at all, instead she was looking for some alternative model system in Fluid dynamics, being of the opinion that the classical Navier-Stokes equations are not quite correct even for Newtonian fluids if the gradient of the velocity is large, and that they should be somehow modified; see also [33] for more comments. Recently, non-Newtonian fluids have been intensively analyzed by many authors and a considerable literature, related to their behavior, rapidly grew-up; for an account of the mathematical aspects of the theory see the book [28] (see also [27] and [5] for an updated list of references and the paper [11] for parabolic systems with non-linear growth). It is clear that for general systems as (1.1), i.e. without additional structure assumptions on $a$, partial $C^{0, \alpha}$-regularity of the spatial gradient $D u$ is the best possible result available, beside an estimate for the Hausdorff dimension of the singular set (the closed subset outside which $D u$ is Hölder continuous). Indeed, even in the elliptic case, solutions to general elliptic systems of the form $-\operatorname{div} a(x, D u)=0$ are not everywhere regular, while estimates for the Hausdorff dimension of the singular set are available (see for instance [26] for a detailed account of the problem in the elliptic case). It is anyway important to note that in the case of the usual Navier-Stokes equations a wider and deeper theory has been developed (see $[6,20,34,35])$.

A new interesting kind of fluids of prominent technological interest has recently emerged: the so called electrorheological fluids. These are special fluids characterized by their ability to change in a dramatic way their mechanical properties when in presence of an external electromagnetic field; for instance they are able to increase their viscosity by a factor 1000 in a few milliseconds. In the context of continuum mechanics these fluids have been modelled as non-Newtonian fluids. The basic studies can be found in the papers [29,30] while the fundamental mathematical analysis for the model can be found in the monograph [31] (see also [32,9]). According to the model proposed by Rajagopal and Růžička, the system governing an electrorheological fluid looks to the naïve eye exactly as the one in (1.1) apart for the coupling with the external electromagnetic field $\underline{\mathbf{E}}$ :

$$
\left\{\begin{array}{l}
\operatorname{curl} \underline{\mathbf{E}}=0, \quad \operatorname{div} \underline{\mathbf{E}}=0, \\
u_{t}-\operatorname{div} a(x, t, \underline{\mathbf{E}}, \mathrm{D}(u))+D \pi=-\operatorname{div}(u \otimes u)+f \quad \text { in } \Omega \times(0, T), \\
\operatorname{div} u=0 .
\end{array}\right.
$$

Here we want to stress the main feature of the previous model: the dependence of $a$ upon $\underline{\mathbf{E}}$ is described via a variable growth exponent, indeed in this case the relation in (1.2) is now substituted by:

$$
a(x, t, \underline{\mathbf{E}}, \mathrm{D}(u)) \approx v(x, t, \underline{\mathbf{E}})\left(1+|\mathrm{D}(u)|^{2}\right)^{\frac{p(\mathbf{E})-2}{2}} \mathrm{D}(u)+\text { terms with similar growth }
$$


that is the exponent $p$ is varying with the electromagnetic field $\underline{\mathbf{E}}$ (this describes the changes of viscosity). Once noted that the system in (1.3) is uncoupled, one can first obtain $\underline{\mathbf{E}}(x, t)$ from Maxwell's equations so that $p \equiv p(x, t)$. This is going to tell us that actually the vector field $a$ exhibits non-standard growth conditions:

$$
c_{1}|F|^{\gamma_{1}}-c_{2} \leqslant a(x, t, F): F \leqslant c_{3}|F|^{\gamma_{2}}+c_{3}, \quad 1<\gamma_{1}<\gamma_{2}<+\infty,
$$

where

$$
\gamma_{1}=\inf p(x, t), \quad \gamma_{2}=\sup p(x, t)
$$

The previous ones, i.e. with a gap between the monotonicity and the growth exponents, are known in the literature as non-standard growth conditions of $(p, q)$ type (in this case $p \equiv \gamma_{1}$ and $\left.q \equiv \gamma_{2}\right)$ and they have been the object of an extensive series of papers starting with the counterexamples of Giaquinta and Marcellini [15,24] and the regularity theory of Marcellini for the scalar case, [25] (see also [10] for an updated list of references). It is interesting to remark that Zhikov used a similar model to describe the behavior of a conductor influenced in a somewhat analogous way by the temperature (see [37]).

The first purpose of this paper is to begin the study of regularity issues for the non-stationary system of electrorheological fluids (1.3) by starting with the model situation of a parabolic system with non-standard growth:

$$
\left\{\begin{array}{l}
u_{t}-\operatorname{div} a(x, t, D u)=0, \\
c_{1}|F|^{p(x, t)}-c_{2} \leqslant a(x, t, F): F \leqslant c_{3}|F|^{p(x, t)}+c_{3}, \quad p(x, t)>1,
\end{array}\right.
$$

in $\Omega \times(0, T)$. Indeed we prove partial $C^{0, \alpha}$ regularity of spatial gradient of weak solutions (defined in a suitable sense and named Energy Solutions; see Definition 2.1) to system (1.5), see Theorem 2.1. We remark that as far as partial $C^{0, \alpha}$ regularity of the spatial gradient is concerned, this is the first result for parabolic systems, under non-standard growth conditions. We remark that even in the scalar case, the literature on the issue is not vast and we mention here the nice paper by Lieberman [22], concerning everywhere regularity of the spatial gradient in the scalar case $N=1$. We also observe that for the sake of brevity and in order to highlight the main ideas, we confined ourselves to the analysis of homogeneous systems but, in principle, the techniques developed here allow to treat more general systems with a non-zero right-hand side, provided this satisfies suitable growth assumptions.

Related regularity results in the stationary case can be found in [2,3] (see also [8,1] for the variational case). Moreover, fluids with viscosity dependence described using non-standard growth conditions have been treated, in the stationary case, in various settings; see for instance $[5,13,14,12,4]$; this paper also offers an approach which is potentially useful to extend such results to the non-stationary case.

Finally we spend a few words about the techniques. In Section 3 we study the problem in cylinders where $p(z)$ has small oscillations ("cylinders of the type $Q_{0}$ "). Here we prove an a priori estimate (Theorem 3.1), ensuring the higher integrability of the spatial gradient in $Q_{0}$; at this stage an interpolation-iteration procedure tailored for parabolic problems plays a central role (Lemmas 3.3 and 3.5). This is first done for a priori regular solutions (Section 3) and then adapted to the original one via approximation (Section 4); here we observe that such an approximation argument works since the peculiar $p(z)$-growth structure of the problem is compatible with the usual convolution (see Appendix A and Lemma 4.1). In Section 5 we consider a blow up procedure exploited only in cylinders of the type $Q_{0}$. The advantage is that we can use the higher integrability to choose a suitable excess functional involving the maximum of $p(z)$ in $Q_{0}$, allowing to overcome the fact that the problem exhibits non-standard growth conditions (the use of such a "maximal excess" allows to treat, in a certain sense, the problem as one with standard polynomial growth). Finally we cover the full cylinder $\Omega \times(0, T)$ with small cylinders of the type $Q_{0}$, and, using the results in Sections 5, we blow up the solution in each of these, thereby proving partial regularity in each $Q_{0}$; the conclusion follows (see the comments in Section 6). 


\section{Statements and notations}

\subsection{Basic notations}

In the following $\Omega$ will denote a bounded domain in $\mathbb{R}^{n}$, and $Q_{T}$ will be the cylinder $\Omega \times(0, T)$. Differentiation with respect to the space variables will be denoted with a comma in lower indices, for example, $\partial f / \partial x_{j}=f_{, j}$. For functions, vectors fields and matrix fields, respectively, we use the differential operators $D f:=(f, j), D v:=\left(v_{i, j}\right)$, $\operatorname{div} A=\left(A_{i j, j}\right)$, all of which are understood in the sense of distributions; differentiation with respect to the time variable will be denoted by $\partial_{t}$ or by ${ }_{t}: \partial_{t} f \equiv f_{t}$. The symbols $L^{p}\left(\Omega ; \mathbb{R}^{n}\right)$ and $W^{1, p}\left(\Omega ; \mathbb{R}^{n}\right)$ stand for the usual Lebesgue and Sobolev spaces, and will be often abbreviated into $L^{p}(\Omega)$ and $W^{1, p}(\Omega)$, respectively (or even $L^{p}$ and $\left.W^{1, p}\right)$. The norm in $L^{p}(\Omega)$ will be abbreviated in $\|\cdot\|_{p, \Omega}$. The spaces $C^{\alpha, \beta}$ are those of functions which are $\alpha$-Hölder continuous in the space variables and $\beta$-Hölder continuous in the time variable. We shall keep the standard notation concerning balls and parabolic cylinders:

$$
\begin{aligned}
& B(x, R):=\left\{y \in \mathbb{R}^{n}:|x-y|<R\right\} \\
& Q(z, R) \equiv Q((x, t), R):=B(x, R) \times] t-R^{2}, t[.
\end{aligned}
$$

They will be simply denoted by $B_{R}$ and $Q_{R}$ respectively when no ambiguity about the center of the ball or the vertex of the cylinder shall arise; also, except when differently specified, all balls (or cylinders) will have the same center (vertex). If $v$ is an integrable function in $Q\left(z_{0}, R\right)$ we shall denote its average by:

$$
(v)_{z_{0}, R}:=\frac{1}{\omega_{n} R^{n+2}} \int_{Q\left(z_{0}, R\right)} v d x d t=\int_{Q\left(z_{0}, R\right)} v d x d t,
$$

where $\omega_{n}$ denotes the measure of $n$-dimensional unit ball in $\mathbb{R}^{n}$; we shall often abbreviate $(v)_{z_{0}, R} \equiv(v)_{R}$ when no confusion about the vertex will arise.

We shall deal with vector fields depending on many variables, for instance $a(x, t, F): \mathbb{R}^{n} \times \mathbb{R} \times \mathbb{R}^{n N} \rightarrow \mathbb{R}^{n N}$; differentiation with respect to the $x$ variable will be denoted by $D_{x}$, as e.g. $D_{x} a$, while differentiation with respect to $F$ will be denoted by $D_{F}$ or simply by $D$, e.g.: $D a \equiv D_{F} a$.

If $A \subset \mathbb{R}^{n}$ and $t \in \mathbb{R}$, we shall denote by $A_{t}$ the layer

$$
A_{t}:=A \times\{t\} .
$$

We recall that the parabolic Hausdorff Measure $\mathcal{P}_{s}$ is defined as follows:

$$
\begin{aligned}
& \mathcal{P}_{s}^{\delta}(F):=\inf \left\{\sum_{i=1}^{\infty} R_{i}^{s}: F \subset \bigcup_{i=1}^{\infty} Q\left(z_{i}, R_{i}\right), R_{i} \leqslant \delta\right\}, \\
& \mathcal{P}_{S}(F):=\sup _{\delta>0} \mathcal{P}_{s}^{\delta}(F) .
\end{aligned}
$$

Finally, in the following, the constant $c$ will simply denote an unspecified, positive quantity, possibly changing from line to line, while only the critical connections will be remarked; more peculiar instances will be denoted by $\tilde{c}, \check{c}$ and so on. In the rest of the paper we shall use Einstein's convention on repeated indices.

\subsection{Systems and energy solutions}

We are given an exponent function $p: z \in Q_{T} \mapsto p(z) \in(1,+\infty)$ which is Lipschitz continuous with respect to the space variables and $\beta / 2$-Hölder continuous with respect to time (in the following $\left.(x, t),\left(x_{0}, t_{0}\right) \in Q_{T}\right)$, that is:

$$
\left|p(x, t)-p\left(x_{0}, t_{0}\right)\right| \leqslant L\left(\left|x-x_{0}\right|+\left|t-t_{0}\right|^{\beta / 2}\right), \quad \beta \in(0,1]
$$


moreover we shall assume that:

$$
\frac{2 n}{n+2}<\gamma_{1} \leqslant p(z) \leqslant \gamma_{2}<+\infty .
$$

Let us observe that the previous lower bound on $\gamma_{1}$ and, consequently, on $p(z)$, is typical in the theory of nonlinear parabolic systems and equations.

We shall consider a vector field $a:(z, F) \in Q_{T} \times \mathbb{R}^{n N} \mapsto a(z, F) \in \mathbb{R}^{n N}$; with abuse of notation, if $z \equiv(x, t)$ we shall also denote $a(z, F) \equiv a(x, t, F)$. We assume that the functions $(z, F) \mapsto a(z, F)$ and $(z, F) \mapsto D a(z, F)$ are continuous in $Q_{T} \times \mathbb{R}^{n N}$ and the following growth and ellipticity conditions are satisfied:

$$
\begin{aligned}
& |a(z, F)| \leqslant L\left(1+|F|^{2}\right)^{(p(z)-1) / 2}, \\
& |D a(z, F)| \leqslant L\left(1+|F|^{2}\right)^{(p(z)-2) / 2}, \\
& \operatorname{Da}(z, F) A: A \geqslant L^{-1}\left(1+|F|^{2}\right)^{(p(z)-2) / 2}|A|^{2},
\end{aligned}
$$

for all $z \in Q_{T}$ and $A, F \in \mathbb{R}^{n N}$ where $1 \leqslant L<+\infty$. According to (2.1), we shall assume the following continuity property, clearly modeled on the behaviuor of $a(z, F)=\left(1+|F|^{2}\right)^{(p(z)-2) / 2} F$ :

$$
\begin{aligned}
\left|a(z, F)-a\left(z_{0}, F\right)\right| \leqslant & L\left[\left(1+|F|^{2}\right)^{(p(z)-1) / 2}+\left(1+|F|^{2}\right)^{\left(p\left(z_{0}\right)-1\right) / 2}\right] \\
& \times \log (2+|F|)\left(\left|x-x_{0}\right|+\left|t-t_{0}\right|^{\beta / 2}\right)
\end{aligned}
$$

for any $z \equiv(x, t)$ and $z_{0} \equiv\left(x_{0}, t_{0}\right)$ and for all $F \in \mathbb{R}^{n N}$ where $\beta$ is as in (2.1).

We are now ready to give the definition of Energy Solution:

Definition 2.1. A function $u \in L^{2}\left(Q_{T} ; \mathbb{R}^{N}\right)$ is an Energy Solution to the parabolic system:

$$
\partial_{t} u-\operatorname{div} a(z, D u)=0
$$

iff

$$
\int_{Q_{T}}|D u|^{p(z)} d z<+\infty
$$

and

$$
\int_{Q_{T}} u \partial_{t} w-a(z, D u): D w d z=0
$$

for any $w \in C_{0}^{\infty}\left(Q_{T} ; \mathbb{R}^{N}\right)$.

\subsection{Main result}

Our main regularity result concerning weak solutions is the following:

Theorem 2.1. Let $u$ be an energy solution of the system (2.7) under the assumptions (2.1)-(2.6). There is an open subset $Q_{T}^{0} \subset Q_{T}$ such that $D u \in C^{\beta_{1}, \beta_{1} / 2}\left(Q_{T}^{0}\right)$ with any exponent $\beta_{1}<\beta$ and $\left|Q_{T} \backslash Q_{T}^{0}\right|=0$.

The statement of the previous theorem requires some comments; we see that no bound has been imposed on the size of the oscillations of the function $p(z)$, that is on the number $\gamma_{2}-\gamma_{1}$. This is particularly relevant when referred to the context of electrorheological fluids. Indeed the size of the number $\gamma_{2}-\gamma_{1}$ keeps into account the possible excursions of the function $p(\underline{\mathbf{E}})$, that is of the viscosity of the fluid, when the electromagnetic field $\underline{\mathbf{E}}$ 
changes. Of course, the larger the number $\gamma_{2}-\gamma_{1}$, the larger the class of fluids that the model is able to cover. Therefore we allow all possible large values of $\gamma_{2}-\gamma_{1}$. This is also seemingly in contrast to what is usually done in the framework of non-standard growth conditions of $(p, q)$ type (see [25], for instance) for elliptic systems, where a bound on the quantity $q-p$ in terms of that ratio $q / p$ is usually assumed. Indeed it turns out (see [10]) that:

$$
\frac{q}{p} \leqslant 1+\frac{\beta}{n}
$$

is in general a necessary condition for regularity, where $\beta$, as in (2.6), is the Hölder continuity exponent with respect to the $x$-variable. In particular $q / p \rightarrow 1$ when $n \rightarrow+\infty$. The point here is that we fully use the $p(z)$ growth structure of the problem.

\section{An a priori estimate}

In this section we shall prove an a priori estimate that will be used in the sequel. This estimate is concerned with solutions to a perturbed system, with "standard" polynomial growth and ellipticity conditions.

First of all let us recall some notations we shall keep for the rest of the section. In the following $Q_{0}:=$ $B\left(\overline{x_{0}}, R\right) \times\left(t_{1}, t_{2}\right)$ will be a fixed cylinder, with $B\left(\overline{x_{0}}, R\right) \Subset \Omega$. Then we shall put:

$$
p_{2}:=\sup _{Q_{0}} p(z) \leqslant \gamma_{2}, \quad p_{1}:=\inf _{Q_{0}} p(z) \geqslant \gamma_{1}>\frac{2 n}{n+2}
$$

while $q>\max \left\{p_{2}, 2\right\}$ will be a number specified below. Moreover $\varepsilon \in(0,1)$ and $v^{\varepsilon} \in C^{0}\left(\left[t_{1}, t_{2}\right] ; L_{\mathrm{loc}}^{2}(\Omega)\right) \cap$ $L^{q}\left(t_{1}, t_{2} ; W_{\text {loc }}^{1, q}\left(\Omega ; \mathbb{R}^{N}\right)\right)$ will be the solution to the perturbed parabolic system

$$
\int_{Q_{0}}-v^{\varepsilon} \partial_{t} w+a_{\varepsilon}\left(z, D v^{\varepsilon}\right): D w d z=0, \quad \forall w \in C_{0}^{\infty}\left(Q_{0} ; \mathbb{R}^{N}\right),
$$

where

$$
a_{\varepsilon}(z, F):=a(z, F)+\varepsilon\left(1+|F|^{2}\right)^{\frac{q-2}{2}} F .
$$

We will assume that the oscillation of $p$ is not too large: precisely, in this section we assume that for some $\alpha>0$

$$
\begin{array}{ll}
p_{2}-p_{1} \leqslant \alpha / 2<\alpha<\frac{2}{(n+2) n} & \text { if } p_{2} \geqslant 2, \\
p_{2}-p_{1} \leqslant \alpha / 2<\alpha<\min \left\{\frac{4}{n^{3}}, \frac{1}{2}\left(\gamma_{1}-\frac{2 n}{n+2}\right)\right\} & \text { if } p_{2}<2,
\end{array}
$$

and we set

$$
q_{0}:=p_{1}+\frac{4}{n} .
$$

We also define the number $q$ according to:

$$
q:= \begin{cases}p_{2}+\alpha & \text { if } p_{2} \geqslant 2, \\ 2+\alpha & \text { if } p_{2}<2,\end{cases}
$$

and therefore in any case, by (3.3),

$$
q>2, \quad q_{0}>q>p_{2} .
$$

The main result of this section is the following a priori estimate for $v^{\varepsilon}$ : 
Theorem 3.1 (A priori estimate). Assume that (3.3) holds for some $\alpha>0$, and let $Q\left(z_{0}, 2 \Gamma\right) \Subset Q_{0}$; then the following estimate holds for the functions $v^{\varepsilon}$ :

$$
\int_{Q\left(z_{0}, \Gamma / 2\right)}\left|D v^{\varepsilon}\right|^{q_{0}} d z \leqslant c \Gamma^{n+2}+c\left(\frac{1}{\Gamma}\right)^{s_{1}}\left(\int_{Q\left(z_{0}, \Gamma\right)}\left|D v^{\varepsilon}\right|^{p_{1}} d z\right)^{s_{2}}
$$

where

$$
s_{1}=\frac{8}{4-\left(q-p_{1}\right)(n+2)} \frac{n+2}{n}, \quad s_{2}=\frac{4-\left(q-p_{1}\right) n}{4-\left(q-p_{1}\right)(n+2)} \frac{n+2}{n}
$$

and the constant $c$ depends upon $\left(n, \gamma_{1}, \gamma_{2}, L, \alpha\right)$ but is independent of $\varepsilon$ and of the solution $v^{\varepsilon}$.

For the sake of simplicity, from now on we shall suppose that, with a clear abuse (and even ambiguity) of notations:

$$
Q_{0} \equiv Q_{T}:=A \times(0, T) ; \quad A:=B\left(\overline{x_{0}}, R\right) .
$$

The estimate will be reached through a series of lemmas. In the first one we derive a suitable Caccioppoli type estimate which differs from the usual one due to the non-standard growth conditions we are assuming.

Lemma 3.1. Let $\varphi, \chi$ be two cut-off functions such that $\varphi \in C_{0}^{\infty}\left(A ; \mathbb{R}^{N}\right), 0 \leqslant \varphi \leqslant 1,|D \varphi| \geqslant 1$ and $\chi \in$ $W^{1, \infty}((0, T)), \chi(0)=0$ and $\partial_{t} \chi \geqslant 0$. Let $Q_{t_{0}}:=A \times\left(0, t_{0}\right)$ and:

$$
\begin{aligned}
& P_{\varepsilon}:=\sum_{i=1}^{n} D a_{\varepsilon}\left(z, D v^{\varepsilon}(z)\right) D v_{, i}^{\varepsilon}(z): D v_{, i}^{\varepsilon}(z), \\
& I_{0}(\varphi, \chi):=\int_{Q_{t_{0}}} \chi \varphi^{2} P_{\varepsilon} d z, \quad J_{0}(\varphi, \chi):=\sup _{0<t<t_{0}} \int_{\Omega} \chi(t) \varphi^{2}(x)\left|D v^{\varepsilon}(x, t)\right|^{2} d x .
\end{aligned}
$$

Then

$$
I_{0}(\varphi, \chi)+J_{0}(\varphi, \chi) \leqslant c \int_{Q_{t_{0}}}\left[\partial_{t} \chi \varphi^{2}+\chi\left(\varphi^{2}+|D \varphi|^{2}\right)\right]\left(1+\left|D v^{\varepsilon}\right|^{2}\right)^{q / 2} d z,
$$

with $c \equiv c\left(n, \gamma_{1}, \gamma_{2}, L, \alpha\right)$ independent of $\left(\varepsilon, t_{0}, \chi, \varphi\right)$ and of the particular solution $v^{\varepsilon}$.

Proof. In the following we shall make all the computations in some detail since the same procedure will be required later, in step 3 from Lemma 5.1. It turns out that (recall that $q>2$ ):

$$
\left\{\begin{array}{l}
\int_{\widetilde{Q}}\left(1+\left|D v^{\varepsilon}\right|^{2}\right)^{\frac{p(z)-2}{2}}\left|D^{2} v^{\varepsilon}\right|^{2} d z \leqslant \int_{\widetilde{Q}}\left(1+\left|D v^{\varepsilon}\right|^{2}\right)^{\frac{q-2}{2}}\left|D^{2} v^{\varepsilon}\right|^{2} d z<+\infty, \\
\int_{\widetilde{Q}}\left|D v^{\varepsilon}\right|^{q} d z<+\infty
\end{array}\right.
$$

for any parabolic subcylinder $\widetilde{Q} \Subset Q_{T}$ (see for instance [21]). For any $f \in L_{\text {loc }}^{1}\left(Q_{T} ; \mathbb{R}^{k}\right)$ and $i \in\{1,2, \ldots, n\}$, and $h \neq 0$ we set (with $\left.\left(x+h e_{i}, t\right) \in Q_{T}\right)$ :

$$
\begin{aligned}
& \tau_{h} f(x, t) \equiv\left(\tau_{h, i} f\right)(x, t):=f\left(x+h e_{i}, t\right)-f(x, t), \\
& \Delta_{h} f(x, t) \equiv\left(\Delta_{h, i} f\right)(x, t):=|h|^{-1}\left(f\left(x+h e_{i}, t\right)-f(x, t)\right),
\end{aligned}
$$

where, as usual, $\left\{e_{i}\right\}$ denotes the standard basis of $\mathbb{R}^{n}$. In the weak formulation (2.9) we replace $w$ by $\tau_{-h} w$, where $0<h<\operatorname{dist}\left(\operatorname{supp} \varphi, \partial Q_{0}\right) / 1000$ in order to get:

$$
\int_{Q_{T}} \tau_{h} u \partial_{t} w-\tau_{h} a_{\varepsilon}(z, D u) D w d z=0
$$


If $\left\{g_{\rho}\right\}$ denotes a family of standard, positive radially symmetric mollifiers, replacing $w$ with $w_{\rho} \equiv w * g_{\rho}$ in the previous equation yields:

$$
\int_{Q_{T}}-\left(\tau_{h} u\right)_{\rho} \partial_{t} w+\left(\tau_{h}\left(a_{\varepsilon}(z, D u)\right)\right)_{\rho} D w d z=0 .
$$

In the last formulation we let the test function $w:=\phi\left(\tau_{h} v^{\varepsilon}\right)_{\rho}$ where $\phi \in C_{0}^{\infty}\left(Q_{0}\right)$ is a non-negative cut-off function; this choice yields, after a simple integration by parts:

$$
\begin{aligned}
& -\frac{1}{2} \int_{Q_{T}} \partial_{t} \phi\left|\left(\tau_{h} v^{\varepsilon}\right)_{\rho}\right|^{2} d z+\int_{Q_{T}} \phi\left(\tau_{h}\left(a_{\varepsilon}\left(z, D v^{\varepsilon}\right)\right)\right)_{\rho}: D\left(\tau_{h} v^{\varepsilon}\right)_{\rho} d z \\
& \quad=-\int_{Q_{T}}\left(\tau_{h}\left(a_{\varepsilon}\left(z, D v^{\varepsilon}\right)\right)\right)_{\rho}: D \phi \otimes\left(\tau_{h} v^{\varepsilon}\right)_{\rho} d z .
\end{aligned}
$$

By (3.9) we can let $\rho \rightarrow 0$ to get:

$$
-\frac{1}{2} \int_{Q_{T}} \partial_{t} \phi\left|\tau_{h} v^{\varepsilon}\right|^{2}+\int_{Q_{T}} \phi \tau_{h} a_{\varepsilon}\left(z, D v^{\varepsilon}\right): D \tau_{h} v^{\varepsilon} d z=-\int_{Q_{T}} \tau_{h} a_{\varepsilon}\left(z, D v^{\varepsilon}\right): D \phi \otimes \tau_{h} v^{\varepsilon} d z .
$$

Now we perform the following choice: $\phi(x, t):=\tilde{\chi}(t) \chi(t) \varphi^{2}(x)$ where $\chi$ and $\varphi$ are as in the statement and $\tilde{\chi}$ is continuous function defined as follows: with $0<t_{0}<T$ and $0<\Delta<T-t_{0}$ we let

$$
\tilde{\chi}(t):= \begin{cases}1 & \text { if } t \leqslant t_{0} \\ \text { affine } & \text { if } t_{0} \leqslant t \leqslant t_{0}+\Delta \\ 0 & \text { if } t_{0}+\Delta \leqslant t\end{cases}
$$

With such a choice of $\phi$, letting $\Delta \rightarrow 0$, since $v^{\varepsilon} \in C^{0}\left(\left[t_{1}, t_{2}\right] ; L_{\text {loc }}^{2}(\Omega)\right)$, we get that for every $t \in(0, T)$ :

$$
\begin{gathered}
\frac{1}{2} \int_{A} \chi(t) \varphi^{2}(x)\left|\tau_{h} v^{\varepsilon}(x, t)\right|^{2} d x+\int_{Q_{t_{0}}} \chi \varphi^{2} \tau_{h} a_{\varepsilon}\left(z, D v^{\varepsilon}\right): D \tau_{h} v^{\varepsilon} d z \\
\quad=-2 \int_{Q_{t_{0}}} \chi \varphi \tau_{h} a_{\varepsilon}\left(z, D v^{\varepsilon}\right): D \varphi \otimes \tau_{h} v^{\varepsilon}+\frac{1}{2} \int_{Q_{t_{0}}} \partial_{t} \chi \varphi^{2}\left|\tau_{h} v^{\varepsilon}\right|^{2} d z .
\end{gathered}
$$

Now we split $\tau_{h} a_{\varepsilon}\left(z, D v^{\varepsilon}\right)$ as follows:

$$
\begin{aligned}
\tau_{h} a_{\varepsilon}\left(z, D v^{\varepsilon}\right)= & {\left[a_{\varepsilon}\left(x+h e_{s}, t, D v^{\varepsilon}\left(x+h e_{s}, t\right)\right)-a_{\varepsilon}\left(x, t, D v^{\varepsilon}\left(x+h e_{s}, t\right)\right)\right] } \\
& +\left[a_{\varepsilon}\left(x, t, D v^{\varepsilon}\left(x+h e_{s}, t\right)\right)-a_{\varepsilon}\left(x, t, D v^{\varepsilon}(x, t)\right)\right] \\
=: & T_{1}(h)+T_{2}(h)
\end{aligned}
$$

using (2.6) we get:

$$
\left|T_{1}(h)\right| \leqslant c|h|\left(1+\left|D u\left(x+h e_{i}\right)\right|^{2}\right)^{\frac{p_{2}-1+\alpha / 4}{2}}
$$

where we used the elementary inequality:

$$
\log \left(2+s^{2}\right) \leqslant c(\alpha)\left(1+s^{2}\right)^{\alpha / 8}
$$

Remark 1. The use of (3.12) is the only point causing the dependence on the constant $\alpha$ in the estimate of Theorem 3.1. 
In order to estimate $T_{2}(h)$ from below, we observe that:

$$
\begin{aligned}
T_{2}(h) D \tau_{h} v^{\varepsilon}: D \tau_{h} v^{\varepsilon} & =\int_{0}^{1} D_{z} a_{\varepsilon}\left(x, t, D v^{\varepsilon}+\theta D \tau_{h} v^{\varepsilon}\right) d \theta D \tau_{h} v^{\varepsilon}: D \tau_{h} v^{\varepsilon} \\
& \geqslant L^{-1} \int_{0}^{1}\left(1+\left|D v^{\varepsilon}+\theta \tau_{h} D v^{\varepsilon}\right|^{2}\right)^{\frac{p(z)-2}{2}} d \theta\left|D \tau_{h} v^{\varepsilon}\right|^{2} \\
& \geqslant c^{-1}\left(1+\left|D v^{\varepsilon}(x, t)\right|^{2}+\left|D v^{\varepsilon}\left(x+h e_{i}, t\right)\right|^{2}\right)^{\frac{p(z)-2}{2}}\left|D \tau_{h} v^{\varepsilon}\right|^{2},
\end{aligned}
$$

where $c \equiv c\left(n, L, \gamma_{1}, \gamma_{2}\right)>0$. Finally we introduce a further notation:

$$
B(h):=\left[1+\left|D v^{\varepsilon}(x, t)\right|^{2}+\left|D v^{\varepsilon}\left(x+h e_{i}, t\right)\right|^{2}\right] .
$$

Using (3.11)-(3.13) in (3.10) we obtain:

$$
\begin{aligned}
& \int_{A} \chi(t) \varphi^{2}(x)\left|\tau_{h} v^{\varepsilon}(x, t)\right|^{2} d x+\int_{Q_{t_{0}}} \chi \varphi^{2} T_{2}(h) D \tau_{h} v^{\varepsilon}: D \tau_{h} v^{\varepsilon} d z \\
& \quad+\int_{Q_{t_{0}}} \chi \varphi^{2}\left[B(h)^{\frac{p(z)-2}{2}}+\varepsilon B(h)^{\frac{q-2}{2}}\right]\left|D \tau_{h} v^{\varepsilon}\right|^{2} d z \\
& \leqslant c \int_{Q_{t_{0}}} \chi \varphi^{2}\left[B(h)^{\frac{p(z)-2}{2}}+\varepsilon B(h)^{\frac{q-2}{2}}\right]\left|D \tau_{h} v^{\varepsilon}\right|\left|\tau_{h} v^{\varepsilon}\right||D \varphi| d z \\
& \quad+c \int_{Q_{t_{0}}} \chi B(h)^{\frac{p_{2}-1+\alpha / 4}{2}}\left[\varphi^{2}\left|D \tau_{h} v^{\varepsilon}\right|+2 \varphi\left|D \varphi \| \tau_{h} v^{\varepsilon}\right|\right]|h| d z+c \int_{Q_{t_{0}}} \partial_{t} \chi \varphi^{2}\left|\tau_{h} v^{\varepsilon}\right|^{2} d z
\end{aligned}
$$

We estimate the terms coming from $T_{1}(h)$ (that is the fifth integral of the previous inequality) in the following way (using that $|D \varphi| \geqslant 1$ ):

$$
\begin{aligned}
& \int_{Q_{t_{0}}} \chi B(h)^{\frac{p_{2}-1+\alpha / 4}{2}}\left[\varphi^{2}\left|D \tau_{h} v^{\varepsilon}\right|+2 \varphi|D \varphi|\left|\tau_{h} v^{\varepsilon}\right|\right]|h| d z \\
& \quad \leqslant \sigma \int_{Q_{t_{0}}} \chi \varphi^{2} B(h)^{\frac{p(z)-2}{2}}\left|D \tau_{h} v^{\varepsilon}\right|^{2} d z+C_{\sigma} \int_{Q_{t_{0}}} \chi \varphi^{2} B(h)^{\frac{p(z)+\alpha}{2}}|h|^{2} d z+c \int_{Q_{t_{0}}} \chi \varphi|D \varphi|^{2} B(h)^{\frac{q-1}{2}}\left|\tau_{h} v^{\varepsilon}\right||h| d z
\end{aligned}
$$

Observe that in the previous estimate we have made crucial use of the fact that $p_{2}-p_{1} \leqslant \alpha / 2$ (see (3.3)). We remark that in all the previous estimates the constant $c$ only depends on $n, \gamma_{1}, \gamma_{2}, L, \alpha$. Now we plug-in the last estimate in (3.14), using Young inequality to manage for the forth integral of (3.14), choosing $\sigma$ small enough in the previous relation and finally dividing up by $|h|^{2}$ we obtain, using the definition of the number $q$ and the fact that $q>2$ (see (3.5)):

$$
\begin{aligned}
& \sup _{0<t<t_{0}} \int_{A} \chi(t) \varphi^{2}(x)\left|\Delta_{h} v^{\varepsilon}(x, t)\right|^{2} d x+\int_{Q_{t_{0}}} \chi \varphi^{2} T_{2}(h) D \Delta_{h} v^{\varepsilon}: D \Delta_{h} v^{\varepsilon} d z \\
& \quad \leqslant c \int_{Q_{t_{0}}} \chi \varphi|D \varphi|^{2}\left[B(h)^{\frac{q-2}{2}}\left|\Delta_{h} v^{\varepsilon}\right|^{2}+B(h)^{\frac{q-1}{2}}\left|\Delta_{h} v^{\varepsilon}\right|\right]+\varphi^{2}\left[\chi+\partial_{t} \chi\right] B(h)^{\frac{q}{2}} d z
\end{aligned}
$$

and the conclusion follows just letting $h \rightarrow 0$, by (3.9). 
Lemma 3.2. Let $\varphi_{1} \in C_{0}^{\infty}\left(A ; \mathbb{R}^{N}\right)$ and $\chi_{1} \in W^{1, \infty}((0, T))$ be two non-negative cut-off functions such that $\chi_{1}(0)=0$ and $\partial_{t} \chi_{1} \geqslant 0$; let $0<t_{0}<T$. Then:

$$
\begin{aligned}
& \int_{Q_{t_{0}}} \chi_{1} \varphi_{1}^{2}\left|D v^{\varepsilon}\right|^{q_{0}} d z \\
& \quad \leqslant c\left[\sup _{t<t_{0}, \chi_{1}(t) \neq 0} \int_{\operatorname{spt} \varphi_{1}}\left(1+\left|D v^{\varepsilon}(x, t)\right|^{2}\right) d x\right]^{2 / n}\left[I_{0}\left(\varphi_{1}, \chi_{1}\right)+\int_{Q_{t_{0}}} \chi_{1}\left|D \varphi_{1}\right|^{2}\left|D v^{\varepsilon}\right|^{p_{1}} d z\right]
\end{aligned}
$$

with $c \equiv c\left(n, \gamma_{1}, \gamma_{2}, L\right)$ independent of $\left(t_{0}, \chi_{1}, \varphi_{1}, \varepsilon\right)$.

Proof. Let us introduce the following function:

$$
H:=\frac{1}{1+\alpha_{0}}\left|D v^{\varepsilon}\right|^{1+\alpha_{0}}, \quad \alpha_{0}:=\frac{q_{0}-2}{2}
$$

and note that $\alpha_{0}>0$ by (3.6). Using Sobolev's embedding theorem, one has the inequality:

$$
\begin{aligned}
\int_{A_{t}} \varphi_{1}^{2} H^{2} d x & \leqslant c\left(\int_{\Omega_{t}}\left|D\left(\varphi_{1} H\right)\right|^{\frac{2 n}{n+2}} d x\right)^{\frac{n+2}{n}} \\
& \leqslant c\left(\int_{A_{t}}\left|\varphi_{1} D H\right|^{\frac{2 n}{n+2}} d x\right)^{\frac{n+2}{n}}+c\left(\int_{A_{t}}\left|D \varphi_{1} H\right|^{\frac{2 n}{n+2}} d x\right)^{\frac{n+2}{n}}=: c A_{4}+c A_{5} .
\end{aligned}
$$

By Hölder inequality we get, using also and recalling that from (3.6) and (3.15) it also follows $\left(2 \alpha_{0}+2-p_{1}\right) n / 2=$ 2, we get

$$
\begin{aligned}
A_{4} & =\left[\int_{A_{t}}\left(\varphi_{1}^{2}\left(1+\left|D v^{\varepsilon}\right|^{2}\right)^{\frac{p_{1}-2}{2}}\left|D^{2} v^{\varepsilon}\right|^{2}\right)^{\frac{n}{n+2}}\left(\left|D v^{\varepsilon}\right|^{2 \alpha_{0}}\left(1+\left|D v^{\varepsilon}\right|^{2}\right)^{\frac{2-p_{1}}{2}}\right)^{\frac{n}{n+2}} d x\right]^{\frac{n+2}{n}} \\
& \leqslant\left(\int_{A_{t}} \varphi_{1}^{2}\left(1+\left|D v^{\varepsilon}\right|^{2}\right)^{\frac{p(z)-2}{2}}\left|D^{2} v^{\varepsilon}\right|^{2} d x\right)\left(\int_{\operatorname{spt} \varphi_{1}}\left(1+\left|D v^{\varepsilon}(x, t)\right|^{2}\right) d x\right)^{\frac{2}{n}} .
\end{aligned}
$$

Moreover, again by Hölder inequality and since $\left(1+\alpha_{0}\right)(2 n) /(n+2)=\left(p_{1} n+4\right) /(n+2)$, we gain

$$
\begin{aligned}
A_{5} & =\left(\frac{2}{q_{0}}\right)^{2}\left(\int_{A_{t}}\left(\left|D \varphi_{1}\right|^{2}\left|D v^{\varepsilon}\right|^{p_{1}}\right)^{\frac{n}{n+2}}\left|D v^{\varepsilon}\right|^{\frac{4}{n+2}} d x\right)^{\frac{n+2}{n}} \\
& \leqslant\left(\int_{A_{t}}\left|D \varphi_{1}\right|^{2}\left|D v^{\varepsilon}\right|^{p_{1}} d x\right)\left(\int_{\operatorname{spt} \varphi_{1}}\left|D v^{\varepsilon}(x, t)\right|^{2} d x\right)^{\frac{2}{n}} .
\end{aligned}
$$

Integrating on $\left(0, t_{0}\right)$ via (3.16), (3.17) and (3.18) we conclude the proof:

$$
\begin{aligned}
& \int_{Q_{t_{0}}} \varphi_{1}^{2} \chi_{1}\left|D v^{\varepsilon}\right|^{q_{0}} d z \leqslant c \int_{0}^{t_{0}} \chi_{1}\left(\int_{\operatorname{spt} \varphi_{1}}\left(1+\left|D v^{\varepsilon}(x, t)\right|^{2}\right) d x\right)^{\frac{2}{n}} \times\left(\int_{A_{t}} \varphi_{1}^{2} P_{\varepsilon}+\left|D \varphi_{1}\right|^{2}\left|D v^{\varepsilon}\right|^{p_{1}} d x\right) d t \\
& \quad \leqslant c\left[\sup _{0<t<t_{0}, \chi_{1}(t) \neq 0} \int_{\operatorname{spt} \varphi_{1}}\left(1+\left|D v^{\varepsilon}(x, t)\right|^{2}\right) d x\right]^{\frac{2}{n}}\left[I_{0}\left(\varphi_{1}, \chi_{1}\right)+\int_{Q_{t_{0}}} \chi_{1}\left|D \varphi_{1}\right|^{2}\left|D v^{\varepsilon}\right|^{p_{1}} d z\right] .
\end{aligned}
$$


The next lemma gives a first form of the estimate in Theorem 3.1:

Lemma 3.3. Assume that $\Gamma>0$ is such that the cylinder $Q\left(z_{0}, \Gamma\right) \Subset Q_{T}$. Then:

$$
\int_{Q\left(z_{0}, \rho\right)}\left|D v^{\varepsilon}\right|^{q_{0}} d z \leqslant c\left[\frac{1}{(R-\rho)^{2}} \int_{Q\left(z_{0}, R\right)}\left(1+\left|D v^{\varepsilon}\right|^{q}\right) d z\right]^{\frac{2}{n}+1}
$$

whenever $0<\Gamma / 2 \leqslant \rho<R \leqslant \Gamma$. In (3.19) the constant $c \equiv c\left(n, \gamma_{1}, \gamma_{2}, L, \alpha\right)$ is independent of $\left(z_{0}, \Gamma, \rho, R, \varepsilon\right)$.

Proof. In Lemma 3.2 we choose the cut-off functions as follows: $\varphi_{1}$ is such that $\varphi_{1} \equiv 1$ in $B\left(x_{0}, \rho\right), \varphi_{1} \equiv 0$ out of $B\left(x_{0},(\rho+R) / 2\right)$ and such that $\left|D \varphi_{1}\right| \leqslant c(R-\rho)^{-1}$ in $A$. As for $\chi_{1}$ we let:

$$
\chi_{1}(t):= \begin{cases}0 & \text { if } t_{0}-\left(\frac{R+\rho}{2}\right)^{2} \geqslant t, \\ \frac{t+((R+\rho) / 2)^{2}-t_{0}}{((R+\rho) / 2)^{2}-\rho^{2}} & \text { if } t_{0}-\rho^{2} \geqslant t \geqslant t_{0}-\left(\frac{R+\rho}{2}\right)^{2}, \\ 1 & \text { if } t \geqslant t_{0}-\rho^{2} .\end{cases}
$$

It obviously follows that $\left|\partial_{t} \chi_{1}\right| \leqslant 4 /(R-\rho)^{2}$. With such a choice of $\varphi_{1}$ and $\chi_{1}$ Lemma 3.2 yields:

$$
\begin{aligned}
\int_{Q\left(z_{0}, \rho\right)}\left|D v^{\varepsilon}\right|^{q_{0}} d z \leqslant & c\left[\sup _{t_{0}-((R+\rho) / 2)^{2}<t<t_{0}} \int_{B\left(x_{0},(R+\rho) / 2\right)}\left(1+\left|D v^{\varepsilon}(x, t)\right|^{2}\right) d x\right]^{2 / n} \\
& \times\left[\int_{Q\left(z_{0},(R+\rho) / 2\right)} P_{\varepsilon}(z) d z+\frac{1}{(R-\rho)^{2}} \int_{Q\left(z_{0},(R+\rho) / 2\right)}\left|D v^{\varepsilon}\right|^{p_{1}} d z\right] .
\end{aligned}
$$

Now we turn to Lemma 3.1 and we perform a suitable choice of the test functions $\varphi$ and $\chi$. We choose $\varphi$ in such a way that $\varphi \equiv 1$ in $B\left(x_{0}, \frac{R+\rho}{2}\right), \varphi \equiv 0$ out of $B\left(x_{0}, R\right)$ and $1 \leqslant|D \varphi| \leqslant c(R-\rho)^{-1}$ in $A$. Analogously we pick the following $\chi$ :

$$
\chi(t):= \begin{cases}0 & \text { if } t_{0}-R^{2} \geqslant t \\ \frac{t+R^{2}-t_{0}}{R^{2}-((R+\rho) / 2)^{2}} & \text { if } t_{0}-R^{2} \geqslant t \geqslant t_{0}-\left(\frac{R+\rho}{2}\right)^{2}, \\ 1 & \text { if } t \geqslant t_{0}-\left(\frac{R+\rho}{2}\right)^{2} .\end{cases}
$$

Once again $\left|\partial_{t} \chi\right| \leqslant 4 /(R-\rho)^{2}$. With such a choice it follows from Lemma 3.1 that:

$$
\begin{aligned}
& \int_{Q\left(z_{0},(R+\rho) / 2\right)} P_{\varepsilon}(z) d z+\sup _{t_{0}-((R+\rho) / 2)^{2}<t<t_{0}} \int_{B\left(x_{0},(R+\rho) / 2\right)}\left(1+\left|D v^{\varepsilon}(x, t)\right|^{2}\right) d x \\
& \leqslant c\left[\frac{1}{(R-\rho)^{2}} \int_{Q\left(z_{0}, R\right)}\left(1+\left|D v^{\varepsilon}\right|^{2}\right)^{q / 2} d z\right] .
\end{aligned}
$$

Finally, the lemma follows merging this last bound with the one in (3.20). 
The following is restatement of Lemma 6.1 from [16]:

Lemma 3.4. Let $h:[\Gamma / 2, \Gamma] \rightarrow \mathbb{R}$ be a non-negative bounded function, and let $A, B, \beta_{1} \beta_{2}>0$. Assume that for any $\rho$ and $R$ such that $\Gamma / 2 \leqslant \rho<R \leqslant \Gamma$ :

$$
h(\rho) \leqslant \frac{1}{2} h(R)+\frac{A}{(R-\rho)^{\beta_{1}}}+\frac{B}{(R-\rho)^{\beta_{2}}} .
$$

Then there exists $c \equiv c\left(\beta_{1}, \beta_{2}\right)$, such that:

$$
h(\Gamma / 2) \leqslant c\left(\frac{A}{\Gamma^{\beta_{1}}}+\frac{B}{\Gamma^{\beta_{2}}}\right) .
$$

Lemma 3.5. Assume there are numbers $q_{1} \geqslant 1, \delta>1, \mu \geqslant 0, \gamma \geqslant 1, \theta \in(0,1)$ such that:

$$
\frac{\theta \gamma}{\delta}<1
$$

Assume that $f \in L^{q_{1} \delta}\left(Q\left(z_{0}, \Gamma\right)\right)$ and:

$$
\int_{Q\left(z_{0}, \rho\right)}|f|^{q_{1} \delta} d z \leqslant \frac{c_{1}}{(R-\rho)^{\mu}}\left(\int_{Q\left(z_{0}, R\right)}(1+|f|)^{q_{1}} d z\right)^{\gamma}
$$

for all $\rho$ and $R$ such that $\Gamma / 2 \leqslant \rho<R<\Gamma$. Then there is a constant $c \equiv c\left(c_{1}, q_{1}, \delta, \mu, \gamma, n, \theta\right)$ such that:

$$
\int_{Q\left(z_{0}, \Gamma / 2\right)}|f|^{q_{1} \delta} d z \leqslant c \Gamma^{(n+2) \gamma-\mu}+\frac{c}{\Gamma^{\frac{\delta \mu}{\delta-\gamma \theta}}}\left(\int_{Q\left(z_{0}, \Gamma\right)}|f|^{\frac{q_{1}(1-\theta) \delta}{\delta-\theta}} d z\right)^{\frac{\gamma(\delta-\theta)}{\delta-\gamma \theta}} .
$$

Proof. Using Hölder inequality we have:

$$
\int_{Q\left(z_{0}, R\right)}|f|^{q_{1}} d z \leqslant\left(\int_{Q\left(z_{0}, R\right)}|f|^{q_{1} \delta} d z\right)^{\frac{\theta}{\delta}}\left(\int_{Q\left(z_{0}, R\right)}|f|^{q_{1} \frac{(1-\theta) \delta}{\delta-\theta}} d z\right)^{\frac{\delta-\theta}{\delta}} .
$$

Therefore, using (3.22) and the previous inequality, it follows that:

$$
\begin{aligned}
\int_{Q\left(z_{0}, \rho\right)}|f|^{q_{1} \delta} d z & \leqslant \frac{c}{(R-\rho)^{\mu}}\left[\Gamma^{(n+2) \gamma}+\left(\int_{Q\left(z_{0}, R\right)}|f|^{q_{1}} d z\right)^{\gamma}\right] \\
& \leqslant \frac{c \Gamma^{(n+2) \gamma}}{(R-\rho)^{\mu}}+\frac{c}{(R-\rho)^{\mu}}\left(\int_{Q\left(z_{0}, R\right)}|f|^{q_{1} \delta} d z\right)^{\frac{\theta \gamma}{\delta}}\left(\int_{Q\left(z_{0}, R\right)}|f|^{q_{1} \frac{(1-\theta) \delta}{\delta-\theta}} d z\right)^{\frac{(\delta-\theta) \gamma}{\delta}} .
\end{aligned}
$$

By (3.22) we can apply Young inequality to get:

$$
\begin{aligned}
\int_{Q\left(z_{0}, \rho\right)}|f|^{q_{1} \delta} d z \leqslant & \frac{1}{2} \int_{Q\left(z_{0}, R\right)}|f|^{q_{1} \delta} d z+\frac{c \Gamma^{(n+2) \gamma}}{(R-\rho)^{\mu}} \\
& +\tilde{c}\left(\frac{1}{R-\rho}\right)^{\frac{\delta \mu}{\delta-\gamma \theta}}\left(\int_{Q\left(z_{0}, \rho\right)}|f|^{\frac{q_{1} \delta(1-\theta)}{\delta-\theta}} d z\right)^{\frac{\gamma(\delta-\theta)}{\delta-\gamma \theta}} .
\end{aligned}
$$

Finally the assertion follows applying Lemma 3.4 with the choice: 


$$
\begin{aligned}
& A:=c \Gamma^{(n+2) \gamma}, \quad B:=c\left(\int_{Q\left(z_{0}, \Gamma\right)}|f|^{\frac{q_{1} \delta(1-\theta)}{\delta-\theta}} d z\right)^{\frac{\gamma(\delta-\theta)}{\delta \theta-\gamma \theta}}, \\
& h(s):=\int_{Q\left(z_{0}, s\right)}|f|^{q_{1} \delta} d z, \quad \beta_{1}:=\mu, \quad \beta_{2}:=\frac{\delta \mu}{\delta-\gamma \theta} .
\end{aligned}
$$

Remark 2. The constant and the quantities appearing in the previous lemma exhibit the following critical behavior:

$$
\lim _{\theta \gamma / \delta \rightarrow 1} c=+\infty
$$

this appears after using Young inequality in (3.24) in order to get (3.25), more precisely the constant $\tilde{c}$ in (3.25) becomes unbounded as $\theta \gamma / \delta \rightarrow 1$. Therefore, when repeatedly applying this lemma, we shall take care that $\theta \gamma / \delta$ stays uniformly bounded away from one, with respect to the parameters involved in the proof.

Proof of Theorem 3.1. We want to apply Lemma 3.5 in the context of Lemma 3.3, therefore in Lemma 3.5 we perform the following choice of the various quantities involved:

$$
f:=\left|D v^{\varepsilon}\right|, \quad \gamma:=(n+2) / n, \quad \mu:=2(n+2) / n, \quad q_{1}:=q
$$

and finally

$$
\delta:=\frac{p_{1}+4 / n}{q} .
$$

Now, as suggested by the statement, we are going to distinguish between the cases $p_{2}<2$ and $p_{2} \geqslant 2$ checking in both cases that the choice of the previous quantities allows to apply Lemma 3.5.

Case $p_{2}<2$. In this case, since $p_{1}>\frac{2 n}{n+2}$ we first obtain that $\delta$ stays uniformly bounded away from 1 with respect to all the parameters involved: $\left(L, p_{1}, p_{2}, \varepsilon\right)$. Indeed:

$$
\delta \geqslant \frac{p_{1}+4 / n}{2+4 / n^{3}}>\left(\frac{2 n}{n+2}+\frac{4}{n}\right)\left(2+\frac{4}{n^{3}}\right)^{-1}>1 .
$$

Let us define $\theta$ as the solution to the equation:

$$
\delta \frac{q(1-\theta)}{\delta-\theta}=p_{1},
$$

i.e.:

$$
\theta=\frac{\delta\left(q-p_{1}\right)}{\delta q-p_{1}}=\frac{n\left(q-p_{1}\right) \delta}{4} .
$$

Then we check, in order to apply Lemma 3.5, that $\theta \in(0,1)$. Indeed, it turns out that also $\theta$ stays bounded away from 1 (from above, this time) uniformly with respect to all the parameters involved; using in turn (3.29) and (3.3) it follows:

$$
\begin{aligned}
0<\theta & =\frac{n\left(q-p_{1}\right)}{4} \frac{p_{1}+4 / n}{2+\alpha} \\
& \leqslant \frac{n}{4}\left(2-\gamma_{1}+\alpha\right) \frac{p_{1}+4 / n}{2} \\
& =\frac{n}{4}\left[2-\frac{2 n}{n+2}+\left(\alpha-\left(\gamma_{1}-\frac{2 n}{n+2}\right)\right)\right] \frac{p_{1}+4 / n}{2}=: \bar{\theta} \\
& <\frac{n}{4}\left(2-\frac{2 n}{n+2}\right) \frac{2+4 / n}{2}=1 .
\end{aligned}
$$


It remains to check condition (3.21) that is:

$$
\begin{aligned}
\frac{\theta \gamma}{\delta} & =\frac{n\left(q-p_{1}\right)}{4} \frac{n+2}{n} \\
& \leqslant \frac{n+2}{4}\left(2+\alpha-\gamma_{1}\right) \\
& =\frac{n+2}{4}\left[2-\frac{2 n}{n+2}+\left(\alpha-\left(\gamma_{1}-\frac{2 n}{n+2}\right)\right)\right]=: \bar{b} \\
& <\frac{n+2}{4}\left(2-\frac{2 n}{n+2}\right)=1
\end{aligned}
$$

We observe that we have used the fact that $\gamma_{1}>\frac{2 n}{n+2}$ via (3.3). So, keeping into account (3.28), we are now able to apply Lemma 3.5 obtaining (3.7). The precise values of $s_{1}$ and $s_{2}$ can now be inferred by looking at Lemma 3.5 and the relations included in (3.27), (3.28) and (3.29).

Remark 3. Here we somehow trace back the dependence of the constants in the previous argumentation. We observe that the distance $1-\bar{b}$ is critical for the constant appearing in (3.21), see also Remark 3 . Since $\bar{b}<1$, then $\theta \gamma / \delta$ stays bounded away from 1 . This quantity, indeed, critically depends on $\gamma_{1}-\frac{2 n}{n+2}$ in the sense that:

$$
\lim _{\gamma_{1} \rightarrow 2 n /(n+2)} 1-\frac{\theta \gamma}{\delta}=0
$$

Consequently, the constant in Theorem 3.1 blows up when $\gamma_{1} \rightarrow \frac{2 n}{n+2}$. Therefore assuming that $\gamma_{1}>\frac{2 n}{n+2}$ we have that the crucial quantity appearing in (3.21) stays uniformly bounded away from 1: $\theta \gamma / \delta<\bar{b}<1$ with $\bar{b}$ depending on $\left(n, \gamma_{1}, \gamma_{2}, L\right)$ but not on $\varepsilon$ and $\alpha$ which, in turn, can be fixed a priori depending only on $n$ and the distance $\gamma_{1}-\frac{2 n}{n+2}$ (in the case $p_{2}<2$ ). Finally observe how also the exponents $s_{1}$ and $s_{2}$ critically depend on the distance $1-\theta \gamma / \delta$; indeed it turns out that:

$$
s_{1}=\frac{\mu}{1-\theta \gamma / \delta}, \quad s_{2}=\frac{\gamma(1-\theta / \delta)}{1-\theta \gamma / \delta} .
$$

Case $p_{2} \geqslant 2$. As before we observe that $\delta$ stays bounded away from 1 :

$$
\delta=\frac{p_{1}+4 / n}{p_{2}+\alpha}=1+\frac{4 / n-\alpha-\left(p_{2}-p_{1}\right)}{p_{2}+\alpha}>1+\frac{4 / n-4 /(n(n+2))}{\gamma_{2}+2 /(n(n+2))}>1,
$$

where we used both inequalities in $(3.3)_{1}$. Then we check that $\theta$, again defined by $(3.28)$, still belongs to $(0,1)$ and is uniformly bounded away from 1 . Let us observe that:

$$
q-p_{1}=\left(p_{2}+\alpha\right)-p_{1} \leqslant 2 \alpha<\frac{4}{(n+2) n}<\frac{4}{n+2} .
$$

Using (3.29) and (3.31) it follows that:

$$
\begin{aligned}
0<\theta & =\frac{n\left(q-p_{1}\right) \delta}{4} \leqslant \frac{\delta}{n+2} \\
& =\frac{p_{1}+4 / n}{q(n+2)} \leqslant \frac{1}{n+2}\left(1+\frac{4}{n q}\right) \\
& <\frac{1}{n+2}\left(1+\frac{4}{n}\right) \leqslant \frac{3}{4},
\end{aligned}
$$


where, of course, we used the fact that $n \geqslant 2$. Again we have to check (3.21); using (3.29) and (3.31):

$$
\frac{\theta \gamma}{\delta} \leqslant \frac{n\left(q-p_{1}\right)}{4} \frac{n+2}{n} \leqslant \frac{n+2}{n} \frac{4 n}{(n+2) 4 n} \leqslant \frac{1}{2},
$$

and the proof is concluded applying again Lemma 3.5 as in the previous case.

A side benefit of Theorem 3.1 is the following higher differentiability result.

Theorem 3.2. Under the assumptions of Theorem 3.1

$$
\int_{Q\left(z_{0}, \Gamma / 4\right)}\left(1+\left|D v^{\varepsilon}\right|^{2}\right)^{\frac{p(z)-2}{2}}\left|D^{2} v^{\varepsilon}\right|^{2} d z \leqslant c\left(n, \gamma_{1}, \gamma_{2}, L, s_{1}, \Gamma\right)\left(\int_{Q\left(z_{0}, \Gamma\right)} 1+\left|D v^{\varepsilon}\right|^{p_{1}} d z\right)^{s_{2}} .
$$

Proof. It suffices to combine estimates (3.7) and (3.8), choosing suitable cut-off functions $\varphi$ and $\chi$ between $B\left(x_{0}, \Gamma / 4\right)$ and $B\left(x_{0}, \Gamma / 2\right)$ and between $\left[t_{0}-(\Gamma / 4)^{2}, t_{0}\right)$ and $\left[t_{0}-(\Gamma / 2)^{2}, t_{0}\right)$, respectively.

\section{An approximation procedure}

We shall keep here the notations introduced in the previous section. In the following we shall develop an approximation procedure aimed to establishing the estimate contained in Theorem 3.1 not only for the functions $v^{\varepsilon}$, but directly for the original solution $u$. The proof of this assertion will be achieved via an approximation argument; a main point is that the usual smoothing procedure by convolution behaves nicely when the exponent $p(z)$ is Hölder continuous (see Appendix A).

The main result of the section is the following (local) higher integrability result, extending the one of Theorem 3.1:

Theorem 4.1. The results and the estimates stated in Theorems 3.1 and 3.2 hold for any Energy Solution $u$ to system (2.7) under the assumptions (2.1)-(2.6). Moreover if $Q_{0}:=B\left(\overline{x_{0}}, R\right) \times\left(t_{1}, t_{2}\right) \Subset Q_{T}$ is as in Section 3, then:

$$
\left\{\begin{array}{l}
D u \in L_{\mathrm{loc}}^{q_{0}}\left(Q_{0} ; \mathbb{R}^{n N}\right), \\
u \in L_{\mathrm{loc}}^{\underline{p}}\left(t_{1}, t_{2} ; W_{\mathrm{loc}}^{2, \underline{p}}\left(B\left(\overline{x_{0}}, R\right) ; \mathbb{R}^{N}\right)\right) \quad \text { with } \underline{p}:=\min \left\{p_{1}, 2\right\} \\
\left(1+|D u|^{2}\right)^{\frac{p(z)-2}{2}}\left|D^{2} u\right|^{2} \in L_{\mathrm{loc}}^{1}\left(Q_{0}\right)
\end{array}\right.
$$

We start with a smoothing procedure, letting:

$$
u_{\gamma}(z):=\int_{Q_{T}} \omega_{\gamma}(\bar{z}-z) u(\bar{z}) d \bar{z},
$$

with $\gamma \in(0,1), \omega_{\gamma}(z)=\omega_{\gamma}^{1}(x) \omega_{\gamma}^{2}(t)$ where $\omega_{\gamma}^{1} \in C_{0}^{\infty}\left(\mathbb{R}^{n}\right)$ is a standard mollifier in $\mathbb{R}^{n}$ i.e.: $\omega_{\gamma}^{1} \geqslant 0$, supp $\omega_{\gamma}^{1} \subset B(0, \gamma)$ and $\int_{\mathbb{R}^{n}} \omega_{\gamma}^{1}(x) d x=1$; as for $\omega_{\gamma}^{2}$ we first let:

$$
\omega_{1}^{2}(t):= \begin{cases}0 & \text { if } 0 \leqslant t \\ c \exp \left(\frac{1}{(t+1) t}\right) & \text { if }-1<t<0 \\ 0 & \text { if } t \leqslant-1\end{cases}
$$


where constant in the previous definition is such that $\int_{\mathbb{R}} \omega_{1}^{2}(t) d t=1$. Then we define, as usual, $\omega_{\gamma}^{2}(t):=$ $\gamma^{-2} \omega_{1}^{2}\left(t / \gamma^{2}\right)$, thus spt $\omega_{\gamma}^{2} \Subset\left(-\gamma^{2}, 0\right)$ and $\int_{\mathbb{R}} \omega_{\gamma}^{2}(t) d t=1$.

Now, let us consider the cylinder $Q_{0}:=B\left(\overline{x_{0}}, R\right) \times\left(t_{1}, t_{2}\right) \equiv B_{0} \times\left(t_{1}, t_{2}\right) \Subset Q_{T}$ from the statement of Theorem 4.1. With $\gamma \in(0,1)$ we consider an increasing continuous function $\varepsilon:[0,1] \rightarrow \mathbb{R}^{+}$such that $\varepsilon(\gamma) \rightarrow 0$ when $\gamma \rightarrow 0$ and:

$$
\lim _{\gamma \rightarrow 0} \varepsilon(\gamma) \int_{Q_{0}}\left|(D u)_{\gamma}\right|^{q} d z=0
$$

(it suffices to take e.g.

$$
\varepsilon(\gamma):=\frac{1}{1+\gamma^{-2}+\left(\int_{Q_{0}}\left|(D u)_{\gamma}\right|^{q} d z\right)^{2}}
$$

with $\gamma>0$ ).

Keeping fixed our Energy Solution $u$, we look for a function $w_{\gamma}: Q_{0} \rightarrow \mathbb{R}^{N}$ with the following properties:

$$
\begin{aligned}
& \left\{\begin{array}{l}
w_{\gamma} \in C\left(\left[t_{1}, t_{2}\right] ; L^{2}\left(B_{0} ; \mathbb{R}^{N}\right)\right) \cap L^{q}\left(t_{1}, t_{2} ; W_{0}^{1, q}\left(B_{0} ; \mathbb{R}^{N}\right)\right), \\
\partial_{t} w_{\gamma} \in L^{\frac{q}{q-1}}\left(t_{1}, t_{2} ; W^{-1, \frac{q}{q-1}}\left(B_{0} ; \mathbb{R}^{N}\right)\right),
\end{array}\right. \\
& w_{\gamma}\left(\cdot, t_{1}\right)=0, \\
& \int_{Q_{0}}\left[-w_{\gamma} \partial_{t} W+a_{\varepsilon(\gamma)}\left(z, D u_{\gamma}+D w_{\gamma}\right)\right]: D W d z=\int_{Q_{0}}(a(z, D u))_{\gamma}: D W d z \quad \forall W \in C_{0}^{\infty}\left(Q_{0} ; \mathbb{R}^{n}\right), \\
& \frac{1}{2}\left\|w_{\gamma}(\cdot, t)\right\|_{L_{2}\left(B_{0}\right)}^{2}+\int_{B_{0} \times\left(t_{1}, t\right)} a_{\varepsilon(\gamma)}\left(z, D u_{\gamma}+D w_{\gamma}\right): D w_{\gamma} d z \\
& \quad \leqslant \int_{B_{0} \times\left(t_{1}, t\right)}(a(z, D u))_{\gamma}: D w_{\gamma} d z \quad \text { for a.e. } t \in\left[t_{1}, t_{2}\right] .
\end{aligned}
$$

We used the notation introduced in the previous section, that is:

$$
a_{\varepsilon(\gamma)}(z, F)=a(z, F)+\varepsilon(\gamma)\left(1+|F|^{2}\right)^{\frac{q-2}{2}} F,
$$

and the exponent $q$ satisfies (3.6).

We remark that for a fixed Energy Solution $u$ and a fixed $\gamma>0$ the existence of a solution $w_{\gamma}$ with the properties stated above is a well established fact [21]. From now on $\gamma$ will abbreviate a sequence of positive numbers $\left\{\gamma_{n}\right\}_{n} \geqslant 3$ such that $\gamma_{n} \rightarrow 0$; from time to time we shall pass to a subsequence that will be still denoted by $\gamma$. We shall assume that $0<\gamma \leqslant \gamma_{0}:=(1 / 1000) \min \left\{\operatorname{dist}\left(B_{0}, \partial \Omega\right), t_{1}, \sqrt{t_{1}}\right\}$.

Lemma 4.1. If $w_{\gamma}$ is a solution to problem (4.3)-(4.6), then:

$$
\begin{aligned}
& \sup _{t_{1}<t<t_{2}}\left\|w_{\gamma}(\cdot, t)\right\|_{L_{2}\left(B_{0}\right)}^{2}+\int_{Q_{0}}\left|D v_{\gamma}\right|^{p(z)}+\varepsilon(\gamma)\left|D v_{\gamma}\right|^{q} d z \\
& \leqslant c\left[\int_{Q_{T}} 1+|D u|^{p(z)} d z+\varepsilon(\gamma) \int_{Q_{0}}\left|D u_{\gamma}\right|^{q} d z\right],
\end{aligned}
$$

where $v_{\gamma} \equiv w_{\gamma}+u_{\gamma}$ and the constant $c \equiv c\left(n, \gamma_{1}, \gamma_{2}\right)$ is independent of $\gamma \in(0,1)$.

Proof. From (4.6) it follows that for a.e. $t \in\left(t_{1}, t_{2}\right)$ : 


$$
\begin{aligned}
& \frac{1}{2}\left\|w_{\gamma}(\cdot, t)\right\|_{L_{2}\left(B_{0}\right)}^{2}+\int_{B_{0} \times\left(t_{1}, t\right)} a_{\varepsilon(\gamma)}\left(z, D u_{\gamma}+D w_{\gamma}\right):\left(D u_{\gamma}+D w_{\gamma}\right) d z \\
& \leqslant c \int_{B_{0} \times\left(t_{1}, t\right)}\left[(a(z, D u))_{\gamma}: D w_{\gamma}+a_{\varepsilon(\gamma)}\left(z, D u_{\gamma}+D w_{\gamma}\right): D u_{\gamma}\right] d z .
\end{aligned}
$$

On the other hand, writing

$$
a_{\varepsilon(\gamma)}(z, F): F=\left(a_{\varepsilon(\gamma)}(z, F)-a_{\varepsilon(\gamma)}(z, 0)\right): F+a_{\varepsilon(\gamma)}(z, 0): F
$$

using (2.3)-(2.5) we easily derive also the following estimate:

$$
\begin{aligned}
& \frac{1}{2}\left\|w_{\gamma}(\cdot, t)\right\|_{L_{2}\left(B_{0}\right)}^{2}+\int_{Q_{0}}\left|D v_{\gamma}\right|^{p(z)}+\varepsilon(\gamma)\left|D v_{\gamma}\right|^{q} d z \\
& \leqslant c \int_{Q_{0}} 1+\left|(a(z, D u))_{\gamma}\right|\left|D w_{\gamma}\right|+\left|D v_{\gamma}\right| d z \\
& \quad+c \int_{Q_{0}}\left(\left(1+\left|D v_{\gamma}\right|^{2}\right)^{\frac{p(z)-1}{2}}+\varepsilon(\gamma)\left(1+\left|D v_{\gamma}\right|^{2}\right)^{\frac{q-1}{2}}\right)\left|D u_{\gamma}\right| d z .
\end{aligned}
$$

We recall that, since $p(z)$ is Hölder continuous we have that (see Appendix A):

$$
\begin{aligned}
\int_{Q_{0}}\left|D u_{\gamma}(z)\right|^{p(z)} d x & \leqslant c \int_{Q_{T}} 1+|D u(z)|^{p(z)} d x<+\infty, \\
\int_{Q_{0}}\left|(a(z, D u))_{\gamma}(z)\right|^{\frac{p(z)}{p(z)-1}} d x & \leqslant c \int_{Q_{T}}|a(z, D u(z))|^{\frac{p(z)}{p(z)-1}} d x \\
& \leqslant c \int_{Q_{T}} 1+|D u(z)|^{p(z)} d x<+\infty .
\end{aligned}
$$

Using (4.2), (4.11), (4.12) we can derive (4.8) from (4.10) using Young's inequality and the lemma is finally proved.

Lemma 4.2. If $w_{\gamma}$ is a solution to problem (4.3)-(4.6), then $I(\gamma) \rightarrow 0$, where:

$$
\begin{aligned}
I(\gamma):= & \sup _{t_{1}<t<t_{2}}\left\|w_{\gamma}(\cdot, t)\right\|_{L_{2}\left(B_{0}\right)}^{2}+\int_{Q_{0}}\left(a\left(z, D v_{\gamma}\right)-a\left(z, D u_{\gamma}\right)\right): D w_{\gamma} d z \\
& +\varepsilon(\gamma) \int_{Q_{0}}\left(1+\left|D v_{\gamma}\right|\right)^{\frac{q-2}{2}}\left|D v_{\gamma}\right|^{2} d z .
\end{aligned}
$$

We postpone the proof of the previous lemma to the one of the following corollary:

Lemma 4.3. With the previous notation it follows that, up to extracting a subsequence,

$$
\begin{aligned}
& v_{\gamma} \rightarrow u \quad \text { in } L^{1}\left(Q_{0} ; \mathbb{R}^{N}\right), \\
& D v_{\gamma} \rightarrow D u \text { in } L^{p_{1}}\left(Q_{0} ; \mathbb{R}^{n N}\right) \text { and a.e. in } Q_{0} .
\end{aligned}
$$


Proof. Indeed from Lemma 4.2 it follows that

$$
w_{\gamma} \rightarrow 0 \quad \text { in } L^{2}\left(Q_{0} ; \mathbb{R}^{n}\right) .
$$

Now we observe that by $(2.5)$

$$
\left(a\left(z, F_{2}\right)-a\left(z, F_{1}\right)\right):\left(F_{2}-F_{1}\right) \geqslant \tilde{c}\left(1+\left|F_{2}\right|^{2}+\left|F_{1}\right|^{2}\right)^{\frac{p(z)-2}{2}}\left|F_{2}-F_{1}\right|,
$$

for any $F_{1}, F_{2} \in \mathbb{R}^{n N}$, where the constant $\tilde{c}$ depends only on $L, \gamma_{1}$ and $n$. From this inequality it follows that if $p_{1} \geqslant 2$ then:

$$
\int_{Q_{0}}\left|D w_{\gamma}\right|^{p_{1}} d z \leqslant c \int_{Q_{0}}\left(1+\left|D w_{\gamma}\right|^{2}+\left|D u_{\gamma}\right|^{2}\right)^{\frac{p_{1}-2}{2}}\left|D w_{\gamma}\right|^{2} d z \leqslant c I(\gamma) .
$$

If $p_{1}<2$, instead, we must argue in a different manner, using Hölder inequality:

$$
\begin{aligned}
\int_{Q_{0}}\left|D w_{\gamma}\right|^{p_{1}} d z & =\int_{Q_{0}}\left(\left|D w_{\gamma}\right|^{2}\left(1+\left|D w_{\gamma}\right|^{2}+\left|D u_{\gamma}\right|^{2}\right)^{\frac{p_{1}-2}{2}}\right)^{\frac{p_{1}}{2}}\left(1+\left|D w_{\gamma}\right|^{2}+\left|D u_{\gamma}\right|^{2}\right)^{\frac{2-p_{1}}{2} \frac{p_{1}}{2}} d z \\
& \leqslant\left(\int_{Q_{0}}\left(1+\left|D w_{\gamma}\right|^{2}+\left|D u_{\gamma}\right|^{2}\right)^{\frac{p_{1}-2}{2}}\left|D w_{\gamma}\right|^{2} d z\right)^{\frac{p_{1}}{2}}\left(\int_{Q_{0}}\left(1+\left|D w_{\gamma}\right|^{2}+\left|D u_{\gamma}\right|^{2}\right)^{\frac{p_{1}}{2}} d z\right)^{\frac{2-p_{1}}{2}} \\
& \leqslant c[I(\gamma)]^{\frac{p_{1}}{2}} \rightarrow 0
\end{aligned}
$$

as $\gamma \rightarrow 0$. So in any case we have that $D w_{\gamma} \rightarrow 0$ in $L^{p_{1}}\left(Q_{0} ; \mathbb{R}^{n}\right)$. Finally, keeping into account that $u_{\gamma} \rightarrow u$ strongly in $L_{\text {loc }}^{1}\left(Q_{T} ; \mathbb{R}^{N}\right)$ and $D u_{\gamma} \rightarrow D u$ strongly in $L_{\text {loc }}^{p_{1}}\left(Q_{T} ; \mathbb{R}^{n N}\right)$, the statement follows using (4.13).

Proof of Lemma 4.2. From (4.6) it is possible to derive the following identity:

$$
\begin{aligned}
& \frac{1}{2}\left\|w_{\gamma}(\cdot, t)\right\|_{L^{2}\left(B_{0}\right)}^{2}+\int_{t_{1}}^{t} \int_{B_{0}}\left(a\left(z, D v_{\gamma}\right)-a\left(z, D u_{\gamma}\right)\right): D w_{\gamma} d z+\varepsilon(\gamma) \int_{t_{1}} \int_{B_{0}}\left(1+\left|D v_{\gamma}\right|^{2}\right)^{\frac{q-2}{2}}\left|D v_{\gamma}\right|^{2} d z \\
& \leqslant \varepsilon(\gamma) \int_{t_{1}}^{t} \int_{B_{0}}\left(1+\left|D v_{\gamma}\right|^{2}\right)^{\frac{q-2}{2}} D v_{\gamma}: D u_{\gamma} d z \\
& \quad+\int_{t_{1}}^{t} \int_{B_{0}}\left((a(z, D u))_{\gamma}-a(z, D u)\right): D w_{\gamma} d z \\
& \quad+\int_{t_{1}}^{t} \int_{B_{0}}\left(a(z, D u)-a\left(z, D u_{\gamma}\right)\right): D w_{\gamma} d z
\end{aligned}
$$

for all $t \in\left(t_{1}, t_{2}\right)$ and, therefore:

$$
I(\gamma) \leqslant c\left(I_{1}(\gamma)+I_{2}(\gamma)+I_{3}(\gamma)\right),
$$

where: 


$$
\begin{aligned}
& I_{1}(\gamma):=\varepsilon(\gamma) \int_{Q_{0}}\left(1+\left|D v_{\gamma}\right|^{2}\right)^{\frac{q-2}{2}}\left|D v_{\gamma} \| D u_{\gamma}\right| d z, \\
& I_{2}(\gamma):=\int_{Q_{0}}\left|\left((a(z, D u))_{\gamma}-a(z, D u)\right) \| D w_{\gamma}\right| d z, \\
& I_{3}(\gamma):=\int_{Q_{0}}\left|a(z, D u)-a\left(z, D u_{\gamma}\right)\right|\left|D w_{\gamma}\right| d z .
\end{aligned}
$$

By Hölder inequality we have:

$$
I_{1}(\gamma) \leqslant\left(\varepsilon(\gamma) \int_{Q_{0}} 1+\left|D v_{\gamma}\right|^{q} d z\right)^{\frac{q-1}{q}}\left(\varepsilon(\gamma) \int_{Q_{0}} 1+\left|D u_{\gamma}\right|^{q} d z\right)
$$

and therefore $I_{1}(\gamma) \rightarrow 0$, by (4.2). Now, with $\delta \in(0,1)$ we use Young's inequality in (4.16) to deduce the estimate:

$$
I_{2}(\gamma)+I_{3}(\gamma) \leqslant \delta \int_{Q_{0}}\left|D w_{\gamma}\right|^{p(z)}+\left|D v_{\gamma}\right|^{p(z)} d z+c_{\delta}\left(I_{4}+I_{5}\right),
$$

where this time we have set:

$$
\begin{aligned}
& I_{4}(\gamma):=\int_{Q_{0}}\left|(a(z, D u))_{\gamma}-a(z, D u)\right|^{\frac{p(z)}{p(z)-1}} d z, \\
& I_{5}(\gamma):=\int_{Q_{0}}\left|a(z, D u)-a\left(z, D u_{\gamma}\right)\right|^{\frac{p(z)}{p(z)-1}} d z .
\end{aligned}
$$

Observe that, by the results in Appendix A, it follows that $I_{4}(\gamma)+I_{5}(\gamma) \rightarrow 0$ when $\gamma \rightarrow 0$. Finally the statement follows looking at (4.17) and letting first $\gamma \rightarrow 0$ and then letting $\delta \rightarrow 0$, keeping into account the boundedness result in (4.8) and the choice (4.2) of $\varepsilon(\gamma)$.

Proof of Theorem 4.1. We take a parabolic cylinder $Q\left(z_{0}, 2 \Gamma\right) \Subset Q_{0}\left(z_{0} \equiv\left(x_{0}, t_{0}\right)\right)$ as in Theorem 3.1; then we apply the approximation procedure described above to $Q_{0}$. At this stage we can use the a priori estimate of Theorem 3.1 for each function $v_{\gamma}$. Indeed by (4.5) it follows that $v_{\gamma}$ solves the problem

$$
\int_{Q_{0}}-v_{\gamma} \partial_{t} w+a_{\varepsilon}\left(z, D v_{\gamma}\right): D w d z=0 \quad \forall w \in C_{0}^{\infty}\left(Q_{0} ; \mathbb{R}^{N}\right)
$$

which is of the type in (3.1). Therefore the a priori estimate in (3.7) is valid for $v_{\gamma}$ with a constant $c$ that does not depend on $\gamma$. Using the result of Lemma 4.3 and the lower semicontinuity of integrals it follows:

$$
\begin{aligned}
\int_{Q\left(z_{0}, \Gamma / 2\right)}|D u|^{q_{0}} d z & \leqslant \liminf _{\gamma} \int_{Q\left(z_{0}, \Gamma / 2\right)}\left|D v_{\gamma}\right|^{q_{0}} d z \\
& \leqslant c \Gamma^{n+2}+\lim _{\gamma} c\left(\frac{1}{\Gamma}\right)^{s_{1}}\left(\int_{Q\left(z_{0}, \Gamma\right)}\left|D v_{\gamma}\right|^{p_{1}} d z\right)^{s_{2}} \\
& =c \Gamma^{n+2}+c\left(\frac{1}{\Gamma}\right)^{s_{1}}\left(\int_{Q\left(z_{0}, \Gamma\right)}|D u|^{p_{1}} d z\right)^{s_{2}}
\end{aligned}
$$


and the proof of the higher integrability is finished; now we turn to the higher differentiability part. The estimate of Theorem 3.2 holds uniformly with respect to $\gamma$. From this fact it immediately follows that the sequence $\left\{v_{\gamma}\right\}_{\gamma}$ is bounded in

$$
L \underline{p}\left(t_{0}-\Gamma^{2} / 16, t_{0} ; W^{2}, \underline{p}\left(B\left(x_{0}, \Gamma / 4\right) ; \mathbb{R}^{N}\right)\right), \quad \underline{p}:=\min \left\{p_{1}, 2\right\}
$$

since $D v_{\gamma} \rightarrow D u$ strongly in $L^{p_{1}}\left(Q\left(z_{0}, \Gamma / 4\right) ; \mathbb{R}^{N}\right)$, up to (not relabelled) subsequences we have that $D^{2} v_{\gamma} \rightarrow$ $D^{2} u$ weakly in $L \underline{p}\left(Q_{0} ; \mathbb{R}^{n^{2} N}\right)$. Finally, by well known lower semicontinuity theorems (in fact, $D v_{\gamma}$ converges strongly, $D^{2} v_{\gamma}$ converges weakly and the integrand is convex with respect to the $D^{2} v_{\gamma}$ argument) and the estimate of Theorem 3.2 it follows:

$$
\begin{aligned}
\int_{Q\left(z_{0}, \Gamma / 4\right)}\left(1+|D u|^{2}\right)^{\frac{p(z)-2}{2}}\left|D^{2} u\right|^{2} d z & \leqslant \liminf _{\gamma} \int_{Q\left(z_{0}, \Gamma / 4\right)}\left(1+\left|D v_{\gamma}\right|^{2}\right)^{\frac{p(z)-2}{2}}\left|D^{2} v_{\gamma}\right|^{2} d z \\
& \leqslant c\left(n, \gamma_{1}, \gamma_{2}, L, s_{1}, \Gamma\right)\left(\int_{Q\left(z_{0}, \Gamma\right)} 1+|D u|^{p_{1}} d z\right)^{s_{2}} .
\end{aligned}
$$

From this $(4.1)_{1}$ and $(4.1)_{3}$ follow via a standard covering argument.

\section{A decay estimate}

In this section we consider an Energy Solution $u$ to the system (2.7) in $Q_{0}$, under the assumptions described in Section 2, where $Q_{0} \equiv B\left(\overline{x_{0}}, R\right) \times\left(t_{1}, t_{2}\right) \Subset Q_{T}$ and $Q_{0}$ satisfies all the requirements described in Section 3, in particular those in Theorem 3.1. Therefore we shall suppose that the numbers $p_{1}, p_{2}, q, q_{0}$ and $\alpha$ are exactly as the ones described in (3.3)-(3.5) and (3.6). In this situation we are able to apply Theorem 4.1 (which is actually a consequence of Theorem 3.1) from Section 4 to gain both higher integrability and higher differentiability for the spatial gradient of $u$. Finally we perform a last reduction, since the result of Theorem 4.1 is only local with respect to $Q_{0}$ : up to passing to subcylinders $\widetilde{Q} \Subset Q_{0}$ and proving Propositions 5.1 and 5.2 in $\widetilde{Q}$, we shall suppose that (4.1) hold globally in $Q_{0}$. Summarizing, we get, essentially by Theorem 4.1, that:

$$
\int_{Q_{0}}|D u|^{q_{0}} d z<+\infty, \quad \int_{Q_{0}}\left(1+|D u|^{2}\right)^{\frac{p(z)-2}{2}}\left|D^{2} u\right|^{2}<+\infty .
$$

After such a preliminary discussion we are going to introduce the fundamental quantity, namely an "excess" functional measuring, in an integral way, the oscillations of $D u$ in a small parabolic cylinder $Q\left(z_{0}, R\right)$. We define, for any $Q\left(z_{0}, R\right) \Subset Q_{0}$ :

$$
\begin{aligned}
U\left(z_{0}, R\right):= & U_{1}\left(z_{0}, R\right)+U_{2}\left(z_{0}, R\right), \\
U_{1}\left(z_{0}, R\right):= & \frac{1}{R}\left(f_{Q\left(z_{0}, R\right)}\left|u(z)-(D u)_{z_{0}, R}\left(x-x_{0}\right)-(u)_{z_{0}, R}\right|^{2} d z\right)^{1 / 2} \\
& +\left(f_{Q\left(z_{0}, R\right)}\left|D u(z)-(D u)_{z_{0}, R}\right|^{2} d z\right)^{1 / 2}, \\
U_{2}\left(z_{0}, R\right):= & \left(f_{Q\left(z_{0}, R\right)}\left|D u(z)-(D u)_{z_{0}, R}\right|^{q} d z\right)^{1 / 2} .
\end{aligned}
$$

Observe that $U_{1}\left(z_{0}, R\right)$ makes sense by (5.1), since $q_{0}>q>2$. 
Let us recall that a regular point $z \in Q_{T}$ for the function $u$ is a point such that the gradient $D u$ is Hölder continuous for any exponent $\beta_{1}<\beta$ (with respect to the standard parabolic metric) in a neighborhood of it. A point which is not regular will be called a non-regular point. The following proposition allows to characterize the regular points via the excess functional $U\left(z_{0}, R\right)$. In the following the exponent $\beta$ is the one introduced in (2.1).

Proposition 5.1. Assume that conditions of Section 3 are satisfied with respect to a cylinder $Q_{0}:=B\left(\overline{x_{0}}, R\right) \times$ $\left(t_{1}, t_{2}\right)$. Let $z_{0} \in Q_{0}$ such that there exists a sequence of radii $\left\{R_{k}\right\}_{k \in \mathbb{N}}, R_{k} \searrow 0$, such that

$$
\lim _{k} U\left(z_{0}, R_{k}\right)=0, \quad \sup _{k \in \mathbb{N}}\left|(D u)_{z_{0}, R_{k}}\right|<+\infty .
$$

Then $z_{0}$ is a regular point for $u$.

A straightforward consequence of the previous result is the following local version of Theorem 2.1:

Proposition 5.2. Under the assumptions of Proposition 5.1, there is an open subset $Q^{0} \subset Q_{0}$ such that Du $\in$ $C^{\beta_{1}, \beta_{1} / 2}\left(Q^{0}\right)$ with any exponent $\beta_{1}<\beta$ and $\left|Q_{0} \backslash Q^{0}\right|=0$.

Proposition 5.1 (and therefore Proposition 5.2) can be proved by a more or less standard iteration argument of algebraic nature starting from the following decay estimate for the excess $U\left(z_{0}, R\right)$ (see, for instance, [7]).

Lemma 5.1. Let $u$ be an Energy Solution to system (2.7), with $Q_{0}$ as described above; let $M, \tau$ and $\beta_{1}$ be such that:

$$
0<M<+\infty, \quad 0<\tau<1, \quad 0<\beta_{1}<\beta .
$$

There exists a positive number $\varepsilon \equiv \varepsilon(M, \tau, \beta)$ such that if:

$$
Q\left(z_{0}, R\right) \Subset Q_{0}, \quad\left|(D u)_{z_{0}, R}\right| \leqslant M, \quad U\left(z_{0}, R\right)<\varepsilon
$$

then

$$
U\left(z_{0}, \tau R\right) \leqslant c_{1}(M) \tau\left[U\left(z_{0}, R\right)+R^{\beta_{1}}\right]
$$

where $c_{1}(M)$ is a positive constant depending only on $M$.

Proof. Step 1: blow up. As usual when using blow up techniques, we argue by contradiction, therefore we find a sequence of non-degenerate cylinders $Q\left(z^{k}, R_{k}\right) \Subset Q_{0}\left(\right.$ where $\left.z^{k} \equiv\left(x^{k}, t^{k}\right)\right)$ such that:

$$
\begin{aligned}
& A^{k}:=\int_{Q\left(z^{k}, R_{k}\right)} D u d z, \quad\left|A^{k}\right| \leqslant M, \\
& \varepsilon_{k}:=U\left(z^{k}, R_{k}\right)+R_{k}^{\beta_{1}} \rightarrow 0
\end{aligned}
$$

but nevertheless:

$$
U\left(z^{k}, R_{k}\right)>2 c_{1}(M) \tau \varepsilon_{k},
$$

where the constant $c_{1}(M)$ will be chosen later, see (5.16), and will be the one included in (5.4). We proceed by scaling and introducing the new space-time variables:

$$
y:=\frac{x-x^{k}}{R_{k}}, \quad s:=\frac{t-t^{k}}{R_{k}^{2}}, \quad l \equiv(y, s) \in Q_{1}=B_{1} \times(-1,0)
$$


and the new functions $v^{k}$, defined on $Q_{1}$ according to:

$$
v^{k}(l):=\frac{u(z)-A^{k}\left(x-x^{k}\right)-(u)_{z^{k}, R_{k}}}{\varepsilon_{k} R_{k}} .
$$

With such a notation, the following relations easily follow:

$$
U\left(z^{k}, \tau R_{k}\right)=\varepsilon_{k} V^{k}(\tau), \quad V^{k}(\tau):=V_{1}^{k}(\tau)+V_{2}^{k}(\tau),
$$

where:

$$
\begin{aligned}
V_{1}^{k}(\tau) & :=\frac{1}{\tau}\left(f_{Q(\tau)}\left|v^{k}-\left(D v^{k}\right)_{\tau} y-\left(v^{k}\right)_{\tau}\right|^{2} d l\right)^{1 / 2}+\left(f_{Q(\tau)}\left|D v^{k}-\left(D v^{k}\right)_{\tau}\right|^{2} d l\right)^{1 / 2}, \\
V_{2}^{k}(\tau) & :=\varepsilon_{k}^{\frac{q}{2}-1}\left(f_{Q(\tau)}\left|D v^{k}-\left(D v^{k}\right)_{\tau}\right|^{q} d l\right)^{1 / 2} .
\end{aligned}
$$

Consequently $\left(v^{k}\right)_{1}=0$ and $\left(D v^{k}\right)_{1}=0$. Moreover, from the definition of $\varepsilon_{k}$ :

$$
1=V^{k}(1)+\frac{R_{k}^{\beta_{1}}}{\varepsilon_{k}} \geqslant\left(f_{Q_{1}}\left|v^{k}\right|^{2} d l\right)^{1 / 2}+\left(f_{Q_{1}}\left|D v^{k}\right|^{2} d l\right)^{1 / 2}+\varepsilon_{k}^{\frac{q}{2}-1}\left(f_{Q_{1}}\left|D v^{k}\right|^{q} d l\right)^{1 / 2}
$$

while (5.7) turns to:

$$
V^{k}(\tau)>2 c_{1}(M) \tau
$$

Regarding the functions $v^{k}$, it follows that each one is an Energy Solution to the system:

$$
\int_{Q_{1}}\left(-v^{k} \partial_{s} w+\sigma^{k}: D_{y} w\right) d l=0 \quad \forall w \in C_{0}^{\infty}\left(Q_{1} ; \mathbb{R}^{N}\right),
$$

where we set

$$
\sigma^{k}:=\varepsilon_{k}^{-1}\left[a\left(x^{k}+R_{k} y, t^{k}+R_{k}^{2} s, A^{k}+\varepsilon_{k} D v^{k}\right)-a\left(x^{k}, t^{k}, A^{k}\right)\right]
$$

According to (5.9), since $q>2$, up to not relabelled subsequences:

$$
\begin{array}{ll}
v^{k} \rightarrow v & \text { in } L^{2}\left(Q_{1} ; \mathbb{R}^{N}\right) \\
D v^{k} \rightarrow D v & \text { in } L^{2}\left(Q_{1} ; \mathbb{R}^{n N}\right) \\
\varepsilon_{k}^{\frac{q-2}{q}} v^{k} \rightarrow 0 & \text { in } L^{q}\left(Q_{1} ; \mathbb{R}^{N}\right) \\
\varepsilon_{k}^{\frac{q-2}{q}} D v^{k} \rightarrow 0 & \text { in } L^{q}\left(Q_{1} ; \mathbb{R}^{n N}\right) \\
z^{k} \equiv\left(x^{k}, t^{k}\right) \rightarrow\left(x_{*}, t_{*}\right)=: z_{*} & \text { in } \mathbb{R}^{n} \times \mathbb{R} \\
A^{k} \rightarrow A_{*} & \text { in } \mathbb{R}^{n N} \\
\varepsilon_{k} D v^{k} \rightarrow 0 & \text { a.e. in } Q_{1} \\
\varepsilon_{k}^{q-2}\left|D v^{k}\right|^{q} \rightarrow 0 & \text { a.e. in } Q_{1} .
\end{array}
$$

Without loss generality we can assume that $z_{*} \in Q_{1}$; (let us just comment that by (5.13) 2 it follows that, up to subsequences, $\varepsilon_{k}^{(q-2) / q}\left|D v_{k}\right| \rightarrow 0$ a.e. and therefore (5.13) follows).

Using standard lower semicontinuity results for integral functionals, it follows:
$(v)_{1}=0$,
$(D v)_{1}=0$,
$\left|A_{*}\right| \leqslant M$,
$V(1) \leqslant 1$, 
where for any $0<\tau \leqslant 1$

$$
V(\tau):=\frac{1}{\tau}\left(f_{Q_{\tau}}\left|v-(D v)_{\tau} y-(v)_{\tau}\right|^{2} d l\right)^{1 / 2}+\left(f_{Q_{\tau}}\left|D v-(D v)_{\tau}\right|^{2} d l\right)^{1 / 2} .
$$

Now we are going to let $k$ pass to the limit in the systems (5.11); in the next step we will prove that the limit function $v$ is a solution to the following linear parabolic system with constant coefficients:

$$
\int_{Q_{1}}-v \partial_{s} w+\frac{\partial a}{\partial F}\left(z_{*}, A_{*}\right) D v: D w d l=0 \quad \forall w \in C_{0}^{\infty}\left(Q_{1} ; \mathbb{R}^{N}\right) .
$$

By (2.4) and (2.5) it follows that the following ellipticity conditions are satisfied:

$$
c^{-1}(M, L)|\lambda|^{2} \leqslant \frac{\partial a}{\partial F}\left(z_{*}, A_{*}\right) \lambda \otimes \lambda \leqslant c(M, L)|\lambda|^{2},
$$

for any $\lambda \in \mathbb{R}^{n N}$, where $c(M, L) \equiv c(M)$ is a bounded constant essentially depending on $M$ only. Therefore, taking into account the standard regularity theory for linear parabolic systems with constant coefficients (see [7]), we conclude that the function $v$ is smooth in $Q_{1}$ and that there is a constant $c_{1} \equiv c_{1}(M)$, which from this moment we choose to appear in (5.7), such that:

$$
V(\tau) \leqslant c_{1}(M) \tau V(1) \leqslant c_{1}(M) \tau, \quad 0<\tau<1 .
$$

Now if we prove that

$$
V^{h}(\tau) \rightarrow V(\tau)
$$

this, via (5.10), implies that

$$
V(\tau) \geqslant 2 c_{1}(M) \tau
$$

which contradicts (5.16), and the proof will be finished.

We now prove (5.15), while (5.17) will be proved in step 3 .

Step 2: proof of (5.15). Let us first derive some preliminary estimates. We split the vector field in (5.12) as follows:

$$
\sigma^{k}:=\sigma_{1}^{k}+\sigma_{2}^{k}
$$

where:

$$
\begin{aligned}
\sigma_{1}^{h} & :=\varepsilon_{k}^{-1}\left[a\left(x^{k}+R_{k} y, t^{k}+R_{k}^{2} s, A^{k}+\varepsilon_{k} D v^{k}\right)-a\left(x^{k}, t^{k}, A^{k}+\varepsilon_{k} D v^{k}\right)\right], \\
\sigma_{2}^{k} & :=\varepsilon_{k}^{-1}\left[a\left(z^{k}, A^{k}+\varepsilon_{k} D v^{k}\right)-a\left(z^{k}, A^{k}\right)\right] .
\end{aligned}
$$

We obtain some estimates for these objects that will be useful below. Using (2.6), (3.5), (5.5) and (5.9) we have:

$$
\begin{aligned}
\left|\sigma_{1}^{k}\right| & \leqslant c \varepsilon_{k}^{-1} R_{k}^{\beta}\left(1+\left|A^{k}+\varepsilon_{k} D v^{k}\right|\right)^{\frac{p_{2}-1}{2}} \log \left(2+\left|A^{k}+\varepsilon_{k} D v^{k}\right|\right) \\
& \leqslant c(M, \alpha) R_{k}^{\beta-\beta_{1}}\left(1+\left|\varepsilon_{k} D v^{k}\right|^{q-1}\right) \\
& \leqslant c(M, \alpha) R_{k}^{\beta-\beta_{1}}\left(1+\varepsilon_{k}^{\frac{2(q-1)}{q}} \varepsilon_{k}^{(q-2) \frac{q-1}{q}}\left|D v^{k}\right|^{q-1}\right)
\end{aligned}
$$

so that, by (5.9),

$$
\int_{Q_{1}}\left|\sigma_{1}^{k}\right|^{\frac{q}{q-1}} d l \rightarrow 0
$$


For $\sigma_{2}^{k}$ we have (using a standard algebraic lemma to treat the second integral):

$$
\begin{aligned}
\left|\sigma_{2}^{k}\right| & =\left|\int_{0}^{1} \frac{\partial a}{\partial F}\left(z^{k}, A^{k}+\theta \varepsilon_{k} D v^{k}\right) D v^{k} d \theta\right| \\
& \leqslant c \int_{0}^{1}\left(1+\left|A^{k}+\theta \varepsilon_{k} D v^{k}\right|^{2}\right)^{\frac{p\left(z^{k}\right)-2}{2}} d \theta\left|D v^{k}\right| \\
& \leqslant c(M)\left(1+\left|D v^{k}\right|+\varepsilon_{k}^{q-2}\left|D v^{k}\right|^{q-1}\right) .
\end{aligned}
$$

From the last inequality and (5.18) we also find:

$$
\left|\sigma^{k}\right| \leqslant c(M)\left[1+\left|D v^{k}\right|+\varepsilon_{k}^{q-2}\left|D v^{k}\right|^{q-1}\right]
$$

and, with (5.9) and using the fact that $q>2$, we get

$$
\left|\sigma^{k}\right|^{\frac{q}{q-1}} \leqslant c(M) \text {. }
$$

In order to get (5.15), it is clear that it suffices proving that:

$$
I_{k}:=\int_{Q_{1}} \sigma^{k}: D w d l \rightarrow \int_{Q_{1}} \frac{\partial a}{\partial F}\left(z_{*}, A_{*}\right) D v: D w d l=: I_{\infty},
$$

for any $w \in C_{0}^{\infty}\left(Q_{1} ; \mathbb{R}^{N}\right)$. To this aim we write:

$$
\begin{aligned}
I_{k}= & \int_{Q_{1}} \sigma_{1}^{k}: D w d l+\int_{Q_{1}}\left(\sigma_{2}^{k}-\frac{\partial a}{\partial F}\left(z^{k}, A^{k}\right) D v^{k}\right): D w d l \\
& +\int_{Q_{1}} \frac{\partial a}{\partial F}\left(z^{k}, A^{k}\right) D v^{k}: D w d l=: I_{k}^{1}+I_{k}^{2}+I_{k}^{3} .
\end{aligned}
$$

By (5.19) and (5.13) it follows that $I_{k}^{1} \rightarrow 0$ and $I_{k}^{3} \rightarrow I_{\infty}$. It remains to prove that $I_{k}^{2} \rightarrow 0$. This can be rapidly seen as follows: fix $0<\sigma<1$ and determine, by Egorov theorem and (5.13),$A \subset Q_{1}$ such that $\left|Q_{1} \backslash A\right|<\sigma$ and $\varepsilon_{k} D v^{k} \rightarrow 0$ uniformly in $A$; then break $I_{k}^{2}$ in two pieces according to:

$$
I_{k}^{2}=\int_{Q_{1} \backslash A}(\ldots) d l+\int_{A}(\ldots) d l=: I_{k}^{4}+I_{k}^{5} .
$$

Using the uniform continuity of $D a$ on bounded subsets we conclude that $I_{k}^{5} \rightarrow 0$ (since $D v^{k}$ is bounded in $L^{1}$ and $D w$ is smooth); in order to treat $I_{k}^{4}$ it suffices to observe that, by (5.20) and (5.21):

$$
\begin{aligned}
\left|I_{k}^{4}\right| & \leqslant c(M)\|D w\|_{L^{\infty}} \int_{Q_{1} \backslash A}\left(1+\left|D v^{k}\right|+\varepsilon_{k}^{q-2}\left|D v^{k}\right|^{q-1}\right) d l \\
& \leqslant c(M)\left[\left|Q_{1} \backslash A\right|^{\frac{1}{2}}+\varepsilon_{k}^{\frac{q-2}{q}}\left|Q_{1} \backslash A\right|^{\frac{1}{q}}\left(\int_{Q_{1}} \varepsilon_{k}^{q-2}\left|D v^{k}\right|^{q} d l\right)^{1-\frac{1}{q}}\right]
\end{aligned}
$$

and keeping into account (5.9) and that $q>2$, we achieve that $I_{k}^{4} \rightarrow 0$ letting first $k \rightarrow+\infty$ and then $\sigma \rightarrow 0$.

Step 3: proof of (5.17). 
In the following we shall extract a compactness information from the existence of second spatial derivatives. In order to do so we shall differentiate the system.

Warning. We warn the reader that the following calculations will be, at the beginning, a bit sloppy. Indeed, in order to proceed in a rigorous way, we should use the same procedure (smoothing plus difference quotient arguments) introduced for the proof of Lemma 3.1. This would take too much time, yielding only technical changes, so we shall proceed formally, differentiating the systems rather than taking difference quotients and assuming the existence of $D_{x} a$ rather than (2.6), which is just Lipschitz continuity, therefore the existence a.e. of $D_{x} a$. More precisely we assume that:

$$
\left|D_{x} a(z, F)\right| \leqslant L\left(1+|F|^{2}\right)^{\frac{p(z)-1}{2}} \log (2+|F|) .
$$

This additional assumption can be easily removed with extra technical-routine efforts, either, as mentioned before, following the procedure of Lemma 3.1 or by a simple approximation method based on mollification of $a$ with respect to $x$. The only difference with respect to Lemma 3.1 is that since now we are dealing directly with the original solution, which is, according to definition only a $L^{2}\left(0, T ; L_{\text {loc }}^{2}\left(\Omega ; \mathbb{R}^{N}\right)\right.$ ) function (and not $C^{0}\left((0, T) ; L_{\text {loc }}^{2}\left(\Omega ; \mathbb{R}^{N}\right)\right)$ as the approximating solutions of Lemma 3.1$)$, then, where previously we used "sup", this will be replaced by "ess sup". We shall proceed this way for the sake of brevity.

Accordingly, we consider the following differentiated system:

$$
\int_{Q_{1}}\left[v_{, i}^{k} \partial_{s} w-\sigma_{, i}^{k}: D w\right] d l=0 \quad \forall w \in C_{0}^{\infty}\left(Q_{1} ; \mathbb{R}^{N}\right) .
$$

In the previous formula we take the test function $w=\tilde{\chi} \chi \varphi^{2} v_{, i}^{k}$ where $\tilde{\chi}$ is as in the proof of Lemma (3.1), $\chi \in W_{0}^{1, \infty}((-1,0))$ such that $\partial_{t} \chi(t) \geqslant 0$ and $\chi(t)=0$ in a small right neighborhood of -1 , while $\varphi \in W_{0}^{1, q}\left(B_{1}\right)$ has the property that $|D \varphi| \geqslant 1$; they are usual cut-off functions to be chosen later. As a result of manipulations similar to the ones at the beginning of Lemma 3.1 we obtain:

$$
\begin{gathered}
\underset{-1<s<0}{\operatorname{ess} \sup _{B_{1}}} \int_{\mathcal{B}} \chi(s) \varphi^{2}(y)\left|D v^{k}(y, s)\right|^{2} d y+\int_{Q_{1}} \chi \varphi^{2} P^{k} d l \\
\leqslant c\left(\left|J_{1}\right|+\left|J_{2}\right|+\left|J_{3}\right|\right)+c \int_{Q_{1}} \varphi^{2} \partial_{s} \chi\left|D v^{k}\right|^{2} d l
\end{gathered}
$$

where:

$$
\begin{aligned}
& P^{k}:=\sum_{i=1}^{n} D a\left(x^{k}+R_{k} y, t^{k}+R_{k}^{2} s, A^{k}+\varepsilon_{k} D v^{k}\right) D v_{, i}^{k}: D v_{, i}^{k}, \\
& J_{1}:=-2 \int \sum_{Q_{1}}^{n} \varphi \chi D a\left(x^{k}+R_{k} y, t^{k}+R_{k}^{2} s, A^{k}+\varepsilon_{k} D v^{k}\right) D v_{, i}^{k}: v_{, i}^{k} \otimes D \varphi d l, \\
& J_{2}:=-\frac{R_{k}}{\varepsilon_{k}} \int_{Q_{1}} \sum_{i=1}^{n} \varphi^{2} \chi D_{x_{i}} a\left(x^{k}+R_{k} y, t^{k}+R_{k}^{2} s, A^{k}+\varepsilon_{k} D v^{k}\right): D v_{, i}^{k} d l, \\
& J_{3}:=-2 \frac{R_{k}}{\varepsilon_{k}} \int_{Q_{1}} \sum_{i=1}^{n} \varphi \chi D_{x_{i}} a\left(x^{k}+R_{k} y, t^{k}+R_{k}^{2} s, A^{k}+\varepsilon_{k} D v^{k}\right): v_{, i}^{k} \otimes D \varphi d l .
\end{aligned}
$$


In (5.24) we are going to use the growth and ellipticity conditions stated in the formulas (2.4), (2.5), (5.22), and we estimate, as in (3.12):

$$
\log \left(1+\left|A^{k}+\varepsilon_{k} D v^{k}\right|^{2}\right) \leqslant c(\alpha)\left(1+\left|A^{k}+\varepsilon_{k} D v^{k}\right|^{2}\right)^{\alpha / 4} .
$$

Moreover we shall denote $p\left(x^{k}+R_{k} y, t^{k}+R_{k}^{2} s\right) \equiv p(-)$. Using Cauchy-Schwartz and Young inequalities, (5.25) and the fact that $R_{k} / \varepsilon_{k}$ stays bounded from above, we obtain, with $\sigma \in(0,1)$ :

$$
\begin{aligned}
\underset{-1<s<0}{\operatorname{ess} \sup } & \int_{B_{1}} \chi(s) \varphi^{2}(y)\left|D v^{k}(y, s)\right|^{2} d l+\int_{Q_{1}} \chi \varphi^{2} P^{k} d l \\
& +\int_{Q_{1}} \chi \varphi^{2}\left(1+\left|A^{k}+\varepsilon_{k} D v^{k}\right|^{2}\right)^{\frac{p(-)-2}{2}}\left|D^{2} v^{k}\right|^{2} d l \\
\leqslant & c \int_{Q_{1}}|D \varphi|\left(1+\left|A^{k}+\varepsilon_{k} D v^{k}\right|^{2}\right)^{\frac{p(-)-2}{2}}\left|D v^{k} \| D^{2} v^{k}\right| d l+c \int_{Q_{1}} \partial_{t} \chi \varphi^{2}\left|D v^{k}\right|^{2} d l \\
& +\frac{R_{k}}{\varepsilon_{k}} \int_{Q_{1}} \chi \varphi\left(\varphi\left|D^{2} v^{k}\right|+\left|D \varphi \| D v^{k}\right|\right)\left(1+\left|A^{k}+\varepsilon_{k} D v^{k}\right|^{2}\right)^{\frac{p(-)-1}{4}} \log \left(2+\left|A^{k}+\varepsilon_{k} D v^{k}\right|^{2}\right) d l \\
\leqslant & \sigma \int_{Q_{1}}\left(1+\left|A^{k}+\varepsilon_{k} D v^{k}\right|^{2}\right)^{\frac{p(-)-2}{2}}\left|D^{2} v^{k}\right|^{2} d l+c \int_{Q_{1}} \partial_{t} \chi \varphi^{2}\left|D v^{k}\right|^{2} d l \\
& +c(M, \alpha, \sigma) \int_{Q_{1}} \chi\left(\varphi^{2}+|D \varphi|^{2}\right)\left(1+\left|D v^{k}\right|+\varepsilon_{k}^{q-2}\left|D v^{k}\right|^{q}\right) d l .
\end{aligned}
$$

Let us observe that the right-hand side of the previous inequality stays bounded form above, by (5.13). We first choose $\sigma$ suitably small, then the cut-off functions $\chi$ and $\varphi$ in an appropriate way, and we deduce from the above the following bound:

$$
\begin{aligned}
& \operatorname{ess~sup}_{-((1+\tau) / 2)^{2} \leqslant s \leqslant 0}\left\|D v^{k}(\cdot, s)\right\|_{L^{2}(B((1+\tau) / 2))}^{2}+\int_{Q((1+\tau) / 2)} P^{k} d l \\
& +\int_{Q((1+\tau) / 2)}\left(1+\left|A^{k}+\varepsilon_{k} D v^{k}\right|^{2}\right)^{\frac{p(-)-2}{2}}\left|D^{2} v^{k}\right|^{2} d l \leqslant c(M) c(\tau),
\end{aligned}
$$

where the constants $c(\tau)$ and $c(M)$ blow up as $\tau \nearrow 1$ and $M \nearrow+\infty$, respectively; both constants are independent of $k \in \mathbb{N}$. Now, using (5.26), we prove that:

$$
\left\|\varepsilon_{k}^{\frac{q-2}{q}} D v^{k}\right\|_{L^{q_{0}}(Q((1+\tau) / 2))} \leqslant c(M) c(\tau)
$$

with the same dependence of the constants outlined above. According to the notation introduced in Lemma 3.2, we set:

$$
\alpha_{0}:=\frac{q_{0}-2}{2} \stackrel{(3.6)}{>} 0, \quad \tilde{v}^{k}:=\varepsilon_{k}^{\frac{q-2}{q}} v^{k}, \quad H:=\frac{\left|D \tilde{v}^{k}\right|^{1+\alpha_{0}}}{1+\alpha_{0}}, \quad 1+\alpha_{0}=\frac{1}{2}\left(p_{1}+\frac{4}{n}\right) .
$$

Up to the end of this section, all integrals will be made on $B((1+\tau) / 2)$ or on $Q((1+\tau) / 2)$; since these tend to be unreadable when used as indices, throughout the section we simply write

$$
B:=B\left(\frac{1+\tau}{2}\right), \quad Q:=Q\left(\frac{1+\tau}{2}\right) \text {. }
$$


Then, using also Sobolev embedding theorem we gain the inequalities:

$$
\int_{B} H^{2} d y \leqslant c(\tau)\left[\left(\int_{B}|D H|^{\frac{2 n}{n+2}} d y\right)^{\frac{n+2}{n}}+\left(\int_{B} H d y\right)^{2}\right]
$$

while using Hölder inequality we get:

$$
\left(\int_{B} H d y\right)^{2} \leqslant c\left(\int_{B}\left|D \tilde{v}^{k}\right|^{p_{1}} d y\right)\left(\int_{B}\left|D \tilde{v}^{k}\right|^{\frac{4}{n}} d y\right) .
$$

Since $4 / n \leqslant 2$, estimate (5.26) leads to the following bound, which is uniform with respect to $s \in\left(-\left(\frac{1+\tau}{2}\right)^{2}, 0\right)$ :

$$
\left(\int_{B} H(y, s) d y\right)^{2} \leqslant c(M) c(\tau) \int_{B}\left|D \tilde{v}^{k}(y, s)\right|^{p_{1}} d y,
$$

where the constant in the previous inequality is again independent of $k \in \mathbb{N}$. Summarizing (5.29)-(5.31) and using in a standard way Hölder inequality, we obtain:

$$
\begin{aligned}
\int_{B} H^{2} d y \leqslant & c(M) c(\tau)\left\{\varepsilon_{k}^{\frac{(q-2) q_{0}}{q}}\left[\int_{B}\left(1+\left|A^{k}+\varepsilon_{k} D v^{k}\right|^{2}\right)^{\frac{p_{1}-2}{2}}\left|D^{2} v^{k}\right|^{2} d y\right]\right. \\
& \left.\times\left[\int_{B}\left(1+\left|A^{k}+\varepsilon_{k} D v^{k}\right|^{2}\right)^{\frac{2-p_{1}}{2} \frac{n}{2}}\left|D v^{k}\right|^{\alpha_{0} n} d y\right]^{\frac{2}{n}}+\int_{B}\left|D \tilde{v}^{k}\right|^{p_{1}} d y\right\} \\
\leqslant & c(M) c(\tau)\left\{\int_{B} P^{k} d y\left[\int_{B}\left(1+\left|A^{k}+\varepsilon_{k} D v^{k}\right|^{2}\right)^{\frac{2-p_{1}}{2} \frac{n}{2}}\left|D v^{k}\right|^{\alpha_{0} n-\Gamma}\left|D v^{k}\right|^{\Gamma} d y\right]^{\frac{2}{n}}\right. \\
& \left.+\int_{B}\left|D \tilde{v}^{k}\right|^{p_{1}} d y\right\},
\end{aligned}
$$

where we have set $\Gamma:=n\left(q_{0}-q\right) / q>0$. Now we check that:

$$
\Gamma<2, \quad \Gamma<\alpha_{0} n .
$$

Indeed, as for the first of (5.33), we notice that if $p_{1}<2$ then, since $q>2$ :

$$
n\left(\frac{q_{0}}{q}-1\right)<n\left(\frac{q_{0}}{2}-1\right) \stackrel{(3.4)}{=} \frac{n}{2}\left(p_{1}+\frac{4}{n}-2\right)<2 .
$$

On the other hand if $p_{1} \geqslant 2$ then:

$$
n\left(\frac{q_{0}}{q}-1\right)<n\left(\frac{q_{0}}{p_{1}}-1\right)=\frac{n}{p_{1}} \frac{4}{n}=\frac{4}{p_{1}} \leqslant 2 .
$$

As for the second inequality in (5.33), we observe that:

$$
\Gamma<\alpha_{0} n \Leftrightarrow \frac{q_{0}}{q}-1<\frac{q_{0}}{2}-1 \Leftrightarrow 2<q,
$$

which is true.

By (5.33), using Hölder inequality in (5.32), we get the following estimate, again uniform in $s \in(-((1+$ $\tau)(2)^{2}, 0$ ): 


$$
\begin{aligned}
\int_{B} H^{2} d y \leqslant & c(M) c(\tau)\left\{\int_{B} P^{k} d y\left(\int_{B}\left|D v^{k}\right|^{2} d y\right)^{\frac{\Gamma}{n}}\right. \\
& \left.\times\left[\int_{B}\left(\left(1+\left|A^{k}+\varepsilon_{k} D v^{k}\right|^{2}\right)^{\frac{2-p_{1}}{2} \frac{n}{2}}\left|\varepsilon_{k} D v^{k}\right|^{\alpha_{0} n-\Gamma}\right)^{\frac{2}{2-T}} d y\right]^{\frac{2-\Gamma}{n}}+\int_{B}\left|D \tilde{v}^{h}\right|^{p_{1}} d y\right\} .
\end{aligned}
$$

We are finally ready to prove (5.27). We first observe that $\left(2-p_{1}\right) n / 2+\alpha_{0} n=2$; therefore integrating (5.34) on $\left(-((1+\tau) / 2)^{2}, 0\right)$ we get:

$$
\begin{aligned}
\int_{Q}\left(\varepsilon_{k}^{\frac{q-2}{q}}\left|D v^{k}\right|\right)^{q_{0}} d l & =\int_{Q} H^{2} d l \\
& \stackrel{(5.26)}{\leqslant} c(M) c(\tau)\left[\int_{Q} P^{k} d l+\int_{Q} 1+\varepsilon_{k}^{q-2}\left|D v^{k}\right|^{q} d l\right] \\
\stackrel{(5.13),(5.26)}{\leqslant} & c(M) c(\tau)
\end{aligned}
$$

and (5.27) is completely proved.

Now we observe that if we let $f_{k}:=\varepsilon_{k}^{q-2}\left|D v^{k}\right|^{q}$ then (5.13) $)_{8}$ implies that $f_{k} \rightarrow 0$ a.e. while (5.27) implies that $\left\{f_{k}\right\}$ is bounded in $L^{q_{0} / q}(Q)$ with $q_{0} / q>1$; therefore it follows that

$$
\varepsilon_{k}^{q-2} \int_{Q}\left|D v^{k}\right|^{q} d l \rightarrow 0
$$

so

$$
V_{2}^{k}(\tau) \rightarrow 0
$$

In order to fully prove (5.17) it remains to prove that

$$
V_{1}^{k}(\tau) \rightarrow V(\tau)
$$

To this aim, first we assume that $p_{1} \geqslant 2$. Then it follows from (5.26) that:

$$
\int_{Q}\left|D^{2} v^{k}\right|^{2} d l \leqslant c(M) c(\tau)
$$

In the second case we assume $p_{1}<2$ and, again by (5.26), we have the bound:

$$
\begin{aligned}
\int_{Q}\left|D^{2} v^{k}\right|^{p_{1}} d l \leqslant & \left(\int_{Q}\left(1+\left|A^{k}+\varepsilon_{k} D v^{k}\right|^{2}\right)^{\frac{p_{1}-2}{2}}\left|D^{2} v^{k}\right|^{2} d l\right)^{\frac{p_{1}}{2}} \\
& \times\left(\int_{Q}\left(1+\left|A^{k}+\varepsilon_{k} D v^{k}\right|^{2}\right)^{\frac{p_{1}}{2}} d l\right)^{\frac{2-p_{1}}{2}} \leqslant c(M) c(\tau)
\end{aligned}
$$

with the constants independent of $k$. Using the multiplicative inequality we gain:

$$
\left(\int_{Q}\left|D v^{k}\right|^{\Gamma_{0}} d l\right)^{\frac{1}{\Gamma_{0}}} \leqslant c\left(\int_{Q}\left|D^{2} v^{k}\right|^{p_{1}} d l+\int_{Q}\left|D v^{k}\right|^{p_{1}} d l\right)^{\frac{\tilde{\alpha}}{p_{1}}} \operatorname{essup}_{s \in\left(-((1+\tau) / 2)^{2}, 0\right)}\left(\int_{B}\left|D v^{k}\right|^{2} d y\right)^{\frac{(1-\tilde{\alpha})}{2}},
$$


where

$$
\tilde{\alpha}=n /(n+2), \quad \Gamma_{0}=p_{1}(n+2) / n>2,
$$

the last inequality following directly by the lower bound in (2.2). By (5.26) and (5.38) we obtain yet another bound:

$$
\int_{Q}\left|D v^{k}\right|{ }^{\Gamma_{0}} d l \leqslant c(M) c(\tau) .
$$

Now, in order to gain compactness, we are going to estimate the time derivatives. To do this, let $w \in C_{0}^{\infty}\left(Q_{T} ; \mathbb{R}^{N}\right)$; we first estimate

$$
\begin{aligned}
\left|\int_{Q} \sigma^{k}: D w d l\right| & \leqslant \int_{-((1+\tau) / 2)^{2}}^{0}\|D w(\cdot, s)\|_{L^{\infty}(B)}\left\|\sigma^{k}(\cdot, s)\right\|_{L^{1}(B)} d s \\
& \leqslant c\left\|\sigma^{k}\right\|_{L^{\frac{q}{q-1}}(Q)}\left(\int_{-((1+\tau) / 2)^{2}}^{0}\|D w(\cdot, s)\|_{L^{\infty}(B)}^{q}\right)^{\frac{1}{q}} \\
& \stackrel{(5.21)}{\leqslant} c(M)\left(\int_{-((1+\tau) / 2)^{2}}^{0}\|D w(\cdot, s)\|_{L^{\infty}(B)}^{q}\right)^{\frac{1}{q}} .
\end{aligned}
$$

By the usual Sobolev inequality:

$$
\|D w\|_{L^{\infty}(B)} \leqslant c\|D w\|_{W_{0}^{2, l}(B)}, \quad l>(n+2) / 2,
$$

we deduce from (5.41) and Eq. (5.11), that:

$$
\left\{\partial_{s} v^{k}\right\}_{k} \text { is bounded in } L^{\frac{q}{q-1}}\left(-((1+\tau) / 2)^{2}, 0 ;\left(W_{0}^{2, l}\left(Q ; \mathbb{R}^{N}\right)\right)^{\prime}\right) .
$$

Now, let us consider again separately the previous cases. If $p_{1} \geqslant 2$ then from (5.37) and an Aubin-Lions type compactness result (see for instance [36], Theorem 2.1, chapter 3) it follows that, up to not relabelled subsequences,

$$
D v^{k} \rightarrow D v \quad \text { in } L^{2}\left(Q ; \mathbb{R}^{n N}\right)
$$

If $p_{1}<2$, from (5.38) and again an Aubin-Lions type result, we first obtain that, again up to subsequences,

$$
D v^{k} \rightarrow D v \text { in } L^{p_{1}}\left(Q ; \mathbb{R}^{n N}\right)
$$

and then by (5.40) we get (5.43), again interpolating $L^{2}$ between $L^{p_{1}}$ and $L^{\Gamma_{0}}$, since by (5.39) it follows that $\Gamma_{0}>2$.

In a similar (actually, much easier) way it follows that:

$$
v^{k} \rightarrow v \quad \text { in } L^{2}\left(Q ; \mathbb{R}^{n N}\right) .
$$

We are ready to finish: indeed (5.43), (5.44) imply (5.36), that togehter with (5.35) completely establish (5.17) and the proof of Lemma 5.1 is now complete.

\section{The main result proved}

In this section we are going to prove Theorem 2.1. We explain the way we proceed. All the results developed in Sections 3-5 have been obtained in a particular situation i.e.: in the basic cylinder $Q_{0}$ a special bound (the one 
described before the statement of Theorem 3.1) for the oscillations of the function $p(z)$, measured in terms of the size $p_{2}-p_{1}$, is valid. In order to use these results for the proof of Theorem 2.1 , the first thing we do is reducing to the previous situation: up to passing to a subcylinder compactly contained in $Q_{T}$, we shall cover $Q_{T}$ with a finite number of small subcylinders in which the oscillations of the exponent function $p(z)$ are small enough to meet the conditions of Theorem 3.1. Then we proceed working in each of these subcylinders, blowing up the solution and thereby proving partial regularity of the solution in each subcylinder. The final result then follows by a covering argument.

Proof of Theorem 2.1. We take an increasing sequence of cylinders $Q_{h} \nearrow Q_{T}$ such that $Q_{h} \Subset Q_{T}$. If we prove that the $D u$ is partially regular in each of the $Q_{h}$ we are clearly done, since in each of them the set of non-regular points will be negligible (see also the argument below). Therefore we can reduce ourself to prove the statement of Theorem 2.1 in a single $Q_{h}$; such a cylinder will denoted by $\widetilde{Q}$. We fix $\alpha \equiv \alpha\left(n, \gamma_{1}\right)>0$ according to the restrictions proposed in Section 3, right-hand side inequalities of (3.3), then we determine $\delta \equiv \delta(p(z), \alpha)$ such that:

$$
\left|z_{1}-z_{2}\right| \leqslant \delta \Leftrightarrow\left|p\left(z_{1}\right)-p\left(z_{2}\right)\right|<\alpha / 2
$$

Then we take a finite covering of $\widetilde{Q},\left\{Q_{(k)}\right\}$, with $Q_{(k)} \Subset Q_{T}$, such that, by (6.1):

$$
\operatorname{diam} Q_{(k)}<\delta, \quad \operatorname{osc}_{Q_{(k)}} p(z)<\alpha / 2 \quad \forall k .
$$

Now, each $Q_{(k)}$ is small enough to meet the conditions imposed in Sections 3-5, that is the ones in the left-hand side inequalities of (3.3), and we can apply Proposition 5.2 to each $Q_{(k)}$; therefore there exists an open subset $Q_{(k)}^{0} \subset Q_{(k)}$ such that $D u$ is Hölder continuous in $Q_{(k)}^{0}$ with any exponent $\beta_{1}<\beta$ and $\left|Q_{(k)} \backslash Q_{(k)}^{0}\right|=0$. Then we set

$$
\widetilde{Q}^{0}:=\bigcup_{k} Q_{(k)}^{0}
$$

and we observe that $D u$ is Hölder continuous in $\widetilde{Q}^{0}$ (which is obviously open) with any exponent $\beta_{1}<\beta$ and $\left|\widetilde{Q} \backslash \widetilde{Q}^{0}\right|=0$, thereby proving Theorem 2.1 .

\section{Estimates for the singular set}

For the sake of simplicity, in this section we shall assume (5.22).

Remarks on the Integrability of Solutions. Here we are going to observe that given a parabolic cylinder $Q_{0}$ as considered in Sections 3-5, and therefore with the bounds in (3.3) being in force, the function $\left|D^{2} u\right|+\left|\partial_{t} u\right|$ is in certain Lebesgue space $L_{\text {loc }}^{\mu_{0}}\left(Q_{0}\right)$. Let us recall that by the results in Section 4 (and again up to passing to subcylinders as in Section 5) we have:

$$
\int_{Q_{0}}|D u|^{q_{0}} d z<+\infty, \quad \int_{Q_{0}}\left(1+|D u|^{2}\right)^{\frac{p(z)-2}{2}}\left|D^{2} u\right|^{2}<+\infty .
$$

We introduce the following exponents:

$$
\mu_{1}:=\frac{2\left(p_{1}+4 / n\right)}{2+4 / n}, \quad \mu_{2}:=\frac{2\left(p_{1}+4 / n\right)}{\left(p_{2}-2\right)+\left(p_{1}+4 / n\right)} .
$$

Observe that trivially $\mu_{1}>1$ while by (3.3) we also have $\mu_{2}>1$. Then we let:

$$
\mu_{0}:= \begin{cases}\mu_{1} & \text { if } p_{2}<2, \\ \mu_{2} & \text { if } p_{1} \geqslant 2, \\ \min \left\{\mu_{1}, \mu_{2}\right\} & \text { if } p_{1}<2 \leqslant p_{2} .\end{cases}
$$


In any case $\mu_{0} \leqslant 2$. Now we wish to show that (7.1) implies:

$$
\left|D^{2} u\right|+\left|\partial_{t} u\right| \in L^{\mu_{0}}\left(Q_{0}\right) .
$$

First we remark that:

$$
\left|D^{2} u\right| \in \begin{cases}L_{\mathrm{loc}}^{\mu_{1}}\left(Q_{0}\right) & \text { if } p_{1}<2 \\ L_{\mathrm{loc}}^{2}\left(Q_{0}\right) & \text { if } p_{1} \geqslant 2 .\end{cases}
$$

Indeed, if $p_{1} \geqslant 2$ then, obviously:

$$
\left|D^{2} u\right|^{2} \leqslant\left(1+|D u|^{2}\right)^{\frac{p(z)-2}{2}}\left|D^{2} u\right|^{2} .
$$

If $p_{1}<2$ then also $\mu_{1}<2$ and Young inequality yields:

$$
\begin{aligned}
\left|D^{2} u\right|^{\mu_{1}} & =\left[\left(1+|D u|^{2}\right)^{\frac{p_{1}-2}{4}}\left|D^{2} u\right|\left(1+|D u|^{2}\right)^{\frac{2-p_{1}}{4}}\right]^{\mu_{1}} \\
& \leqslant c\left[\left(1+|D u|^{2}\right)^{\frac{p_{1}-2}{2}}\left|D^{2} u\right|^{2}+\left(1+|D u|^{2}\right)^{\frac{q_{0}}{2}}\right]
\end{aligned}
$$

since $q_{0}=p_{1}+4 / n=\left(2-p_{1}\right) \mu_{1} /\left(2-\mu_{1}\right)$ and (7.3) follows by (7.1). Now we show that:

$$
\left|\partial_{t} u\right| \in \begin{cases}L_{\mathrm{loc}}^{2}\left(Q_{T}\right) & \text { if } p_{2} \leqslant 2 \\ L_{\mathrm{loc}}^{\mu_{2}}\left(Q_{T}\right) & \text { if } p_{2}>2\end{cases}
$$

In order to establish (7.4) we use the equation:

$$
u_{t}=\operatorname{div} a(z, D u)
$$

and the fact that, via Theorem 3.2 and (7.1), it can be differentiated (locally) with respect to the space variables. Using (2.4), (2.6) and (3.12), we obtain:

$$
\begin{aligned}
\left|\partial_{t} u\right| & \leqslant c\left[\left(1+|D u|^{2}\right)^{\frac{p(z)-2}{2}}\left|D^{2} u\right|+\left(1+|D u|^{2}\right)^{\frac{p_{2}-1}{2}} \log \left(1+|D u|^{2}\right)\right] \\
& \leqslant c\left[\left(1+|D u|^{2}\right)^{\frac{p(z)-2}{2}}\left|D^{2} u\right|+\left(1+|D u|^{2}\right)^{\frac{q-1}{2}}\right]
\end{aligned}
$$

where $q$ has been defined in (3.5). Now, consider the case $p_{2} \leqslant 2$. Then since $p(z) \leqslant 2$ it also follows that:

$$
\left|\partial_{t} u\right|^{2} \leqslant c\left[\left(1+|D u|^{2}\right)^{\frac{p(z)-2}{2}}\left|D^{2} u\right|^{2}+1+|D u|^{2(q-1)}\right] .
$$

We must check that $2(q-1) \leqslant q_{0}=p_{1}+4 / n$; recall that $q=2+\alpha$ if $p_{2} \leqslant 2((3.6))$ therefore, using the fact that $2 n /(n+2)<p_{1}$ it suffices to show that $1+\alpha \leqslant n /(n+2)+2 / n$ which follows from the upper bound on $\alpha$ in (3.3). So, by (7.1), it follows that $\left|\partial_{t} u\right| \in L_{\text {loc }}^{2}$ in the case $p_{2} \leqslant 2$. Next, we consider the case $p_{2}>2$. According to (7.6) we have, using Young inequality (observe that when $p_{2}>2$ then $\mu_{2}<2$ ):

$$
\begin{aligned}
\left|\partial_{t} u\right|^{\mu_{2}} & \leqslant c\left[\left(1+|D u|^{2}\right)^{\frac{(p(z)-2) \mu_{2}}{4}}\left(\left(1+|D u|^{2}\right)^{\frac{p(z)-2}{2}}\left|D^{2} u\right|^{2}\right)^{\frac{\mu_{2}}{2}}+1+|D u|^{(q-1) \mu_{2}}\right] \\
& \leqslant c\left[\left(1+|D u|^{2}\right)^{\frac{p(z)-2}{2}}\left|D^{2} u\right|^{2}+(1+|D u|)^{\frac{(p(z)-2) \mu_{2}}{2-\mu_{2}}}+|D u|^{(q-1) \mu_{2}}\right] .
\end{aligned}
$$

In order to use again (7.1) and making all the terms appearing in the right-hand side of (7.7) integrable, we check that

$$
\frac{(p(z)-2) \mu_{2}}{2-\mu_{2}} \leqslant \frac{\left(p_{2}-2\right) \mu_{2}}{2-\mu_{2}} \leqslant q_{0}, \quad(q-1) \mu_{2} \leqslant q_{0} .
$$

The first inequality follows from the definition of $\mu_{2}$. As for the second one:

$$
(q-1) \mu_{2}=\left(p_{2}+\alpha-1\right) \frac{2 q_{0}}{p_{2}-2+q_{0}} \leqslant q_{0},
$$


which is equivalent to $p_{2}-p_{1}+2 \alpha \leqslant 4 / n$ that follows immediately from (3.3); therefore all the quantities appearing on the right-hand side of (7.7) are integrable by (7.1). In turn, by (7.7) we have $\left|\partial_{t} u\right| \in L_{\text {loc }}^{\mu_{2}}\left(Q_{T}\right)$ thereby completing the proof of (7.4). Finally (7.2) follows by (7.3) and (7.4).

We are ready to give a first result for the estimate of the singular set, which is local, in the sense that it gives an estimate on the singular set when we just look at $Q_{0}$ :

Theorem 7.1. Under the previous assumptions on the cylinder $Q_{0}$ suppose that:

$$
q \leqslant \frac{\mu_{0}(n+2)}{n+2-\mu_{0}} \text {. }
$$

Then, with the notation of Proposition 5.2 it follows that for any $\sigma>0$

$$
\mathcal{P}_{n+2-\mu_{0}+\sigma}\left(Q_{0} \backslash Q^{0}\right)=0 .
$$

Proof. From Proposition 5.1 it follows that $Q_{0} \backslash\left(Q_{T}^{0} \cap Q_{0}\right) \subset \Sigma_{0} \cup \Sigma_{1} \cup \Sigma_{2}$ where

$$
\begin{aligned}
& \Sigma_{0}:=\left\{z \in Q_{0}: \limsup _{\rho \rightarrow 0}\left|(D u)_{\rho}\right|=+\infty\right\}, \\
& \Sigma_{1}:=\left\{z \in Q_{0}: \liminf _{\rho \rightarrow 0} U_{1}(z, \rho)>0\right\}, \\
& \Sigma_{2}:=\left\{z \in Q_{0}: \liminf _{\rho \rightarrow 0} U(z, \rho)>0\right\},
\end{aligned}
$$

therefore it suffices to prove that $\mathcal{P}_{n+2-\mu_{0}+\sigma}\left(\Sigma_{i}\right)=0$ for $i \in\{0,1,2\}$ for any $\sigma>0$. For $\Sigma_{2} \cup \Sigma_{2}$, we observe that (7.9) implies the following inequality:

$$
\begin{aligned}
U\left(z_{0}, R\right) \leqslant & R\left(\int_{Q\left(z_{0}, R\right)}\left|D^{2} u\right|^{\mu_{0}}+\left|\partial_{t} u\right|^{\mu_{0}} d z+\right)^{\frac{1}{\mu_{0}}} d z \\
+ & {\left[R\left(f_{Q\left(z_{0}, R\right)}\left|D^{2} u\right|^{\mu_{0}}+\left|\partial_{t} u\right|^{\mu_{0}} d z+\right)^{\frac{1}{\mu_{0}}}\right]^{\frac{q}{2}} . }
\end{aligned}
$$

Nest we recall that if $S \subset Q_{0}$ denotes the set of points $z_{0} \in Q_{0}$ such that:

$$
\limsup _{\rho \rightarrow 0} R^{\left(\mu_{0}-n-2\right)} \int_{Q\left(z_{0}, R\right)}\left|D^{2} u\right|^{\mu_{0}}+\left|\partial_{t} u\right|^{\mu_{0}} d z>0,
$$

from a well known measure density result (first developed in the context of elliptic systems, see [26] for comments and extensions) adapted to the parabolic geometry of rescaled cylinders it follows $\mathcal{P}_{n+2-\mu_{0}}(S)=0$; therefore, by (7.9), (7.10) and the same reduction lemma it follows that $\mathcal{P}_{n+2-\mu_{0}}\left(\Sigma_{1}\right)=\mathcal{P}_{n+2-\mu_{0}}\left(\Sigma_{2}\right)=0$. In a similar way, via the same measure density arguments it is possible to prove that $\mathcal{P}_{n+2-\mu_{0}+\sigma}\left(\Sigma_{0}\right)=0$.

As stated before, the previous theorem provides an estimate which is local, that is, the size of the singular set depends on the peculiar choice of the cylinder $Q_{0}$, via the number $\mu_{0}$. In order to get a global estimate for the singular set we find uniform bounds for $\mu_{0}$. This is the aim of the next theorem.

Remark 4. Before going on with the proof we make some remarks on the possible choice of the number $\alpha$. It is clear from the statement of Theorem 3.1 that the constant $c$ in (3.7) blows up when $\alpha \rightarrow 0$. Anyway the higher integrability exponent $q_{0}$, which is the one of the gradient, does not change directly: it remains linked to $p_{1}$ via the 
same relation: $q_{0}=p_{1}+4 / n$. The only way $q_{0}$ may depend on $\alpha$ appears in the proof of Theorem 2.1 ; there, $p_{1}$ changes with $Q_{(k)}$, which, in that scheme, depends on $\alpha$.

In the following we shall assume, without loss of generality, that:

$$
\frac{2 n}{n+2}<\gamma_{1}<2 \text {. }
$$

Theorem 7.2. Define $\mu>1$ as follows:

$$
\mu:=\min \left\{\frac{2\left(\gamma_{1}+4 / n\right)}{2+4 / n}, \frac{2\left(\gamma_{2}+4 / n\right)}{2 \gamma_{2}-2+4 / n}\right\} \stackrel{(7.11)}{\leqslant} 2,
$$

and suppose that

$$
\gamma_{2}<\frac{\mu(n+2)}{n+2-\mu}
$$

Then, for any $\sigma>0$

$$
\mathcal{P}_{n+2-\mu+\sigma}\left(Q_{T} \backslash Q_{T}^{0}\right)=0 .
$$

Remark 5. Observe that when $\gamma_{1}=\gamma_{2}=p(z)=2$ then $\mu=2$, as for the usual estimates concerning parabolic systems with linear growth (see, for instance, [7]).

Proof of Theorem 7.2. The proof will be achieved using Theorem 7.1. We fix $\sigma>0$ and we observe that it suffices to consider $\sigma$ so small that (7.13) continues to hold with $\mu-\sigma / 2$ in the place of $\mu$ (observe that the function in the right-hand side of (7.13) is increasing with respect to $\mu$ ). Then we can find $\alpha>0$ satisfying the right-hand inequalities in (3.3) and such that:

$$
\gamma_{2}+\alpha<\frac{(\mu-\sigma / 2)(n+2)}{n+2-(\mu-\sigma / 2)} \quad \text { and } \quad \frac{2\left(\gamma_{2}+4 / n\right)}{2 \gamma_{2}-2+4 / n+\alpha}>\mu-\sigma / 2
$$

Now, with respect to such an $\alpha$, we find a covering family of cylinders $\left\{Q_{(k)}\right\}$ as in the proof of Theorem 2.1 , conditions (6.1), (6.2); our aim is to apply the estimate of the singular set stated in Theorem 7.1 in each cylinder $Q_{(k)}$, with respect to the constant $\mu_{0} \equiv \mu_{0}\left(Q_{(k)}\right)$ (note that $\mu_{0}$ depends on the cylinder $Q_{(k)}$ ). Now let us prove that, in each $Q_{(k)}$ :

$$
\mu-\sigma / 2 \leqslant\left\{\begin{array}{ll}
\mu_{1} & \text { if } p_{2}\left(Q_{(k)}\right)<2 \\
\mu_{2} & \text { if } p_{1}\left(Q_{(k)}\right) \geqslant 2 \\
\min \left\{\mu_{1}, \mu_{2}\right\} \equiv \mu_{0} & \text { if } p_{1}\left(Q_{(k)}\right)<2 \leqslant p_{2}\left(Q_{(k)}\right)
\end{array}=\mu_{0}\left(Q_{(k)}\right) .\right.
$$

We prove that the first two inequalities in (7.16) are true for all values of $p_{1}$ and $p_{2}$, therefore the third one follows. The first inequality is trivial since $\mu_{1} \geqslant \mu$ always, it remains to check the second:

$$
\begin{aligned}
\mu_{2} & =\frac{2 q_{0}}{p_{2}-2+q_{0}} \stackrel{(3.3)}{\geqslant} \frac{2 q_{0}}{p_{1}+\alpha-2+q_{0}}=\frac{2 q_{0}}{2 q_{0}-2-4 / n+\alpha} \\
& =1+\frac{2+4 / n-\alpha}{2 q_{0}-2-4 / n+\alpha} \geqslant 1+\frac{2+4 / n-\alpha}{2 p_{2}-2+4 / n+\alpha} \\
& \geqslant 1+\frac{2+4 / n-\alpha}{2 \gamma_{2}-2+4 / n+\alpha}=\frac{2\left(\gamma_{2}+4 / n\right)}{2 \gamma_{2}-2+4 / n+\alpha} \geqslant \mu-\sigma / 2 .
\end{aligned}
$$

It follows that:

$$
q \leqslant \gamma_{2}+\alpha \stackrel{(7.15)}{<} \frac{(\mu-\sigma / 2)(n+2)}{n+2-(\mu-\sigma / 2)} \stackrel{(7.16)}{<} \frac{\mu_{0}(n+2)}{n+2-\mu_{0}}
$$


therefore we can apply Theorem 7.1 and for each $Q_{(k)}$ (using the notation of Theorem 7.1 with $Q_{0} \equiv Q_{(k)}$ and $\left.Q^{0} \equiv Q_{(k)}^{0}\right): D u$ is Hölder continuous in $Q_{(k)}^{0}$ and

$$
\mathcal{P}_{n+2-\mu+\sigma}\left(Q_{(k)} \backslash Q_{(k)}^{0}\right) \leqslant \mathcal{P}_{n+2-\mu_{0}+\sigma / 2}\left(Q_{(k)} \backslash Q_{(k)}^{0}\right)=0 .
$$

The statement finally follows as at the end of Section 6.

\section{Acknowledgement}

This paper has been partially supported by MIUR via the project "Calcolo delle Variazioni" (Cofin 2000) and by CNR via the project "Modelli Variazionali sotto Ipotesi non-Standard". Part of this paper was written while the second author was vising the Steklov Mathematical Institute, St. Petersburg Branch, in July 2001; he would like to thank the members of the Institute for providing a nice environment and in particular N.N. Ural'tseva and A.A. Arkhipova for many pleasant and interesting discussions and for showing kind hospitality.

\section{Appendix A}

Here we provide a justification for the crucial inequalities stated in (4.11) and (4.12). In the following $u$ will be the function from (4.11), (4.12) while $Q_{0}$ will be the cylinder considered in Section 4; for the sake of brevity we assume here that $Q_{0} \equiv Q\left(z_{0}, R\right)$. The arguments can be easily adapted from the ones in [10] and are also a consequence of those developed by Zhikov, but we sketch them here for the sake of the reader. Using Hölder inequality, by the definition of mollification it follows that

$$
\left|D u_{\gamma}(x, t)\right| \leqslant \gamma^{-(n+2) / \gamma_{1}}\|D u\|_{L^{\gamma_{1}}}=: c_{1} \gamma^{-(n+2) / \gamma_{1}} \text {. }
$$

Now let us define (for $\gamma$ suitably small):

$$
p_{\gamma}(x, t):=\inf \{p(y, s):(y, s) \in Q((x, t), 2 \gamma)\}
$$

and $g_{\gamma}(x, t, F):=|F|^{p_{\gamma}(x, t)}$. The Hölder continuity of $p(x, t)$ implies:

$$
\left|p(x, t)-p_{\gamma}(x, t)\right| \leqslant c_{2} \gamma^{\beta}
$$

(with $c_{2}$ independent of $\gamma$ ) and so, by (A.1):

$$
\begin{aligned}
g\left(x, t, D u_{\gamma}(x, t)\right) & =\left|D u_{\gamma}(x, t)\right|^{\left(p(x, t)-p_{\gamma}(x, t)\right)}\left|D u_{\gamma}(x, t)\right|^{p_{\gamma}(x, t)} \\
& \leqslant c_{1} \gamma^{\frac{(n+2)}{\gamma_{1}}\left(p_{\gamma}(x, t)-p(x, t)\right)}\left|D u_{\gamma}(x, t)\right|^{p_{\gamma}(x, t)} \\
& \leqslant c_{1} \gamma^{-\frac{c_{2}(n+2)}{\gamma_{1}} \gamma^{\beta}}\left|D u_{\gamma}(x, t)\right|^{p_{\gamma}(x, t)} \\
& \leqslant c_{3} g_{\gamma}\left(x, t, D u_{\gamma}(x, t)\right) .
\end{aligned}
$$

We remark that the constant $c_{3}$ is independent of $\gamma$. In turn, the definition of $p_{\gamma}(x, t)$ and Jensen inequality give (recall $z \equiv(x, t))$ :

$$
\begin{aligned}
g_{\gamma}\left(x, t, D u_{\gamma}(x, t)\right) \stackrel{\text { Jensen }}{\leqslant} \int_{Q_{R+\gamma}} g_{\gamma}(x, t, D u(\bar{z})) \omega_{\gamma}(\bar{z}-z) d \bar{z} \\
\leqslant \int_{Q_{R+\gamma}} g(\bar{z}, D u(\bar{z})) \omega_{\gamma}(\bar{z}-z) d \bar{z}
\end{aligned}
$$




$$
\begin{aligned}
& =\left(g(\cdot, D u) * \omega_{\gamma}\right)(x, t) \\
& =g(\cdot, D u)_{\gamma}(x, t) .
\end{aligned}
$$

Therefore, combining the last inequality with (A.2) we come up with

$$
g\left(x, t, D u_{\gamma}(x, t)\right) \leqslant g(\cdot, D u)_{\gamma}(x, t)
$$

as a consequence we get (4.11), and the Lebesgue dominated convergence theorem implies that

$$
g\left(x, t, D u_{\gamma}\right) \rightarrow g(x, t, D u) \quad \text { strongly in } L^{1} .
$$

The same argument (considering $a(z, D u)_{\gamma}$ instead of $\left.(D u)_{\gamma}\right)$ applies for (4.12); indeed also $p(z) /(p(z)-1)$ is Hölder continuous and (4.12) follows in the same way. Again Lebesgue dominated convergence theorem implies the assertion (immediately before the proof of Theorem 4.1) $I_{4}(\gamma)+I_{5}(\gamma) \rightarrow 0$.

\section{References}

[1] E. Acerbi, G. Mingione, Regularity results for a class of functionals with nonstandard growth, Arch. Rational Mech. Anal. 156 (2001) $121-140$.

[2] E. Acerbi, G. Mingione, Regularity results for electrorheological fluids: the stationary case, C. R. Acad. Sci. Paris Ser. I 334 (2002) $817-822$.

[3] E. Acerbi, G. Mingione, Regularity results for stationary electro-rheological fluids, Arch. Rational Mech. Anal. 164 (2002) $213-259$.

[4] M. Bildhauer, M. Fuchs, Partial regularity for variational integrals with $(s, \mu, q)$-growth, Calc. Var. Partial Differential Equations 13 (2001) 537-560.

[5] M. Bildhauer, M. Fuchs, Variants of the Stokes problem: the case of anisotropic potentials J. Math. Fluid Mechanics, submitted for publication.

[6] L. Caffarelli, R.V. Kohn, L. Nirenberg, Partial regularity of suitable weak solutions of the Navier-Stokes equations, Comm. Pure Appl. Math. 35 (1982) 771-831.

[7] S. Campanato, On the nonlinear parabolic systems in divergence form. Hölder continuity and partial Hölder continuity of the solutions, Ann. Mat. Pura Appl. (4) 137 (1984) 83-122.

[8] A. Coscia, G. Mingione, Hölder continuity of the gradient of $p(x)$-harmonic mappings, C. R. Acad. Sci. Paris Ser. I 328 (1999) $363-368$.

[9] L. Diening, Theoretical and numerical results for electrorheological fluids, Ph.D. Thesis, University of Freiburg, 2002.

[10] L. Esposito, F. Leonetti, G. Mingione, Sharp regularity for functionals with $(p, q)$ growth, J. Differential Equations, in press.

[11] J. Frehse, G.A. Seregin, Full regularity for a class of degenerated parabolic systems in two spatial variables, Manuscripta Math. 99 (1999) 517-539.

[12] J. Frehse, G.A. Seregin, Regularity of solutions to variational problems of the deformation theory of plasticity with logarithmic hardening, Amer. Math. Soc. Transl. Ser. 2 (1999) 193.

[13] M. Fuchs, G.A. Seregin, Variational Methods for Problems from Plasticity Theory and for Generalized Newtonian Fluids, in: Lecture Notes in Math., vol. 1749, Springer, Berlin, 2000.

[14] M. Fuchs, G.A. Seregin, Variational methods for fluids of Prandtl-Eyring type and plastic materials with logarithmic hardening, Math. Methods Appl. Sci. 22 (1999) 317-351.

[15] M. Giaquinta, Growth conditions and regularity, a counterexample, Manuscripta Math. 59 (1987) $245-248$.

[16] E. Giusti, Direct Methods in the Calculus of Variations, World Scientific Publishing Co., Inc., River Edge, NJ, 2003.

[17] O.A. Ladyzhenskaya, On nonlinear problems of continuum mechanics, in: Proc. Internat. Congr. Math. (Moscow 1966), Nauka, Moscow, 1968, pp. 560-573; English translation in: Amer. Math. Soc. Translation (2) 70 (1968).

[18] O.A. Ladyzhenskaya, New equations for description of motion of viscous incompressible fluids and global solvability of boundary value problems for them, Proc. Steklov Inst. Math. 102 (1967).

[19] O.A. Ladyzhenskaya, On some modifications of the Navier-Stokes equations for large gradient of velocity, Zap. Nauchn. Sem. Leningrad Odtel. Mat. Inst. Steklov (LOMI) 7 (1968) 126-154; English translation in: Sem. Math. V.A. Steklov Math. Inst. Leningrad 7 (1968).

[20] O.A. Ladyzhenskaya, G.A. Seregin, On partial regularity of suitable weak solutions to the three-dimensional Navier-Stokes equations, J. Math. Fluid Mech. 1 (1999) 356-387.

[21] O.A. Ladyzhenskaya, V.A. Solonnikov, N.N. Uraltseva, Linear and Quasilinear Equations of Parabolic Type, in: Translations of Mathematical Monographs, vol. 23, American Mathematical Society, 1967.

[22] G.M. Lieberman, Gradient estimates for a new class of degenerate elliptic and parabolic equations, Ann. Scuola Norm. Sup. Pisa Cl. Sci. (4) 21 (1994) 497-522.

[23] J.-L. Lions, Quelques methodes de resolution des problemes aux limites non lineaires, Gauthier-Villars, Paris, 1969. 
[24] P. Marcellini, Un exemple de solution discontinue d' un probléme variationnel dans le cas scalaire, Preprint Dip. Mat. "U. Dini”, Univ. Firenze, 1987.

[25] P. Marcellini, Regularity and existence of solutions of elliptic equations with $p, q$-growth conditions, J. Differential Equations 90 (1991) $1-30$.

[26] G. Mingione, The singular set of solutions to non-differentiable elliptic systems, Arch. Rational Mech. Anal. 166 (2003) 287-301.

[27] J. Malek, J. Necas, M. Rủžička, On weak solutions to a class of non-Newtonian incompressible fluids in bounded three-dimensional domains: the case $p \geqslant 2$, Adv. Differential Equations 6 (2001) 257-302.

[28] J. Malek, J. Necas, M. Rokyta, M. Růžička, Weak and Measure-Valued Solutions to Evolutionary PDEs, in: Appl. Math. Math. Comp., vol. 13, Chapman-Hall, London, 1996.

[29] K.R. Rajagopal, A.S. Wineman, Flow of electrorheological materials, Acta Mech. 91 (1992) 57-75.

[30] K.R. Rajagopal, M. Růžička, Mathematical modeling of electrorheological materials, Contin. Mech. Thermodyn. 13 (2001) 59-78.

[31] M. Rủžička, Electrorheological Fluids: Modeling and Mathematical Theory, in: Lecture Notes in Math., vol. 1748, Springer, Berlin, 2000.

[32] M. Růžička, Flow of shear dependent electrorheological fluids, C. R. Acad. Sci. Paris Ser. I Math. 329 (1999) 393-398.

[33] G.A. Seregin, Interior regularity for solutions to the modified Navier-Stokes equations, J. Math. Fluid Mech. 1 (1999) $235-281$.

[34] G.A. Seregin, On the number of singular points of weak solutions to the Navier-Stokes equations, Comm. Pure Appl. Math. 54 (2001) 1019-1028.

[35] G.A. Seregin, Sverak V., Navier-Stokes equations with lower bounds on the pressure, Arch. Rational Mech. Anal. 163 (2002) $65-86$.

[36] R. Temam, Navier-Stokes equations. Theory and Numerical Analysis, third ed., in: Studies in Mathematics and its Applications, vol. 2, North-Holland, Amsterdam, 1984.

[37] V.V. Zhikov, Meyers type estimates for solving the nonlinear Stokes system, Differential Equations 33 (1997) 107-114. 